\title{
Versão Intervalar de Métodos Numéricos para a Solução do Problema de Auto-valores de Matrizes
}

\author{
Cleonice Fátima Bracciali
}

orientador: Prof. Odelar Leite Linhares

Dissertação apresentada ao ICMSC-USP como parte dos requisitos para obtenção do título de Mestre em Ciências de Computação e Matemática Computacional. 
ao prof. Odelar $e$

a meus pais 


\section{Agradecimentos}

Ao prof. Odelar Leite Linhares, pelo estímulo e apoio desde a iniciação científica até a concretização deste trabalho, pela felicidade de ter sido sua orientanda e poder presenciar tantos exemplos de sabedoria, humildade, disposição e incentivos constantes.

À profa Eliana Xavier Linhares de Andrade, pela amizade e ótimo convívio, por todas as vezes que prontamente atendeu às minhas dúvidas e pelas inúmeras discussões e contribuições, muito importantes para a realizaçâo deste trabalho.

Aos meus pais, às minhas irmãs Sonia e Ana Maria e toda minha família, por tanto carinho e confiança e também pela compreensão à minha ausência constante.

Ao Arlindo, pelo carinho, companherismo, estímulo e demasiada confiança dedicados a mim desde que nos conhecemos.

Aos meus professores do IBILCE - UNESP - São José do Rio Preto e do ICMSC - USP - São Carlos, pelo estímulo e conhecimentos básicos necessários à minha formação acadêrnica.

A todos os meus amigos que de uma forma ou de outra ajudaramme a percorrer mais esta etapa de vida.

À FAPESP - Fundação de Amparo à Pesquisa do Estado de São Paulo - pelo apoio financeiro que foi decisivo à realização deste trabalho e pelo permanente estímulo a meus programas de estudos e pesquisas, concedendome; desde agosto de 1987 até julho de 1991, bolsas de Injciaçâo Cientifica e de Mestrado.

A todos que direta ou indiretamente colaboraram para a realização deste trabalho. 


\title{
Resumo
}

O objetivo principal deste trabalho é apresentar e analisar métodos numéricos intervalares para a solução do problema de auto-valores de matrizes reais. Serão apresentados métodos intervalares para a limitação dos auto-valores e respectivos auto-vetores de matrizes simétricas (Nickel, Zhiying), e desenvolvidas versões intervalares de métodos numéricos para a determinação do polinômio característico de matrizes (Leverrier-Faddeev, Danilevsky), bem como de métodos numéricos iterativos clássicos (Rutishauser, Francis) para a soluçâo do citado problema.

\begin{abstract}
The main purpose of this thesis is to introduce and analyse interval numerical methods for the solution of the eigenvalue problem of real matrices. Interval methods for bounding the eigenvalues and respective eigenvectors of symmetric matrices (Nickel, Zhiying) are presented. Interval versions of numerical methods for determining the characteristic polinomial of matrices (Leverrier-Faddeev, Danilevsky), and those of some classical numerical methods (Rutishauser, Francis) to solve the above mentioned problem are also presented.
\end{abstract}




\section{Conteúdo}

Introdução viii

1 Matemática Intervalar 1

1.1 Um Breve Histórico . . . . . . . . . . . . . . . . . . . 1

1.2 Número Intervalar . . . . . . . . . . . . . . . 3

1.3 Aritmética Intervalar .................. 4

1.4 Vetores e Matrizes Intervalares . . . . . . . . . . . . . . 9

1.5 Espaço Métrico $I(\Re) \ldots \ldots \ldots \ldots$

1.6 Aritmética Intervalar Arredondada . . . . . . . . . . . . . 17

2 Métodos Intervalares para Limitar os Auto-valores e Auto-vetores de Matrizes Simétricas $\quad 23$

2.1 Método Proposto por Nickel . . . . . . . . . . . . . . . 23

2.2 Método Proposto por Zhiying . . . . . . . . . . . . 25

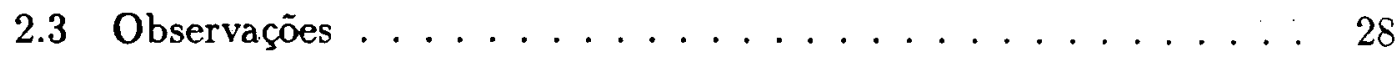

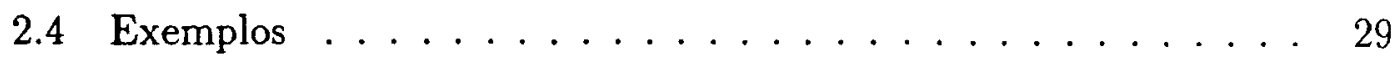


3 Versão Intervalar de Métodos para Determinação dos Auto-valores através do Polinômio Característico da Matriz

3.1 Versão Intervalar de Métodos Numéricos para Determinação do Polinômio Característico de Matrizes . . . . . . . . . . . 30

3.1.1 Método de Leverrier-Faddeev . . . . . . . . . . . . 31

3.1.2 Método de Danilevsky . . . . . . . . . . . . . . 32

3.2 Métodos Intervalares para Determinação de Zeros de Polinômios . 33

3.2.1 Método de Newton-Moore . . . . . . . . . . . . . . . 33

3.2.2 Método de Herzberger $\ldots \ldots \ldots \ldots \ldots$

3.2.3 Método de Hansen $\ldots \ldots \ldots \ldots \ldots \ldots$

3.2.4 Método de Arthur $\ldots \ldots \ldots \ldots \ldots \ldots$

3.3 Observações . . . . . . . . . . . . . . . . . 39

3.4 Exemplos $\ldots \ldots \ldots \ldots \ldots \ldots \ldots \ldots \ldots \ldots$

4 Versão Intervalar de Métodos Iterativos para a Determinação de Auto-valores de Matrizes

4.1 Método LR de Rutishauser . . . . . . . . . . . . . . 50

4.2 Método $\mathrm{QR}$ de Francis $\ldots \ldots \ldots \ldots \ldots \ldots \ldots$

4.3 Observações . . . . . . . . . . . . . . . 55

4.4 Exemplos $\ldots \ldots \ldots \ldots \ldots \ldots \ldots \ldots \ldots$

5 Conclusões $\quad 60$

$\begin{array}{ll}\text { Referências Bibliográficas } & 62\end{array}$ 


\section{Glossário}

$A=$ intervalo real

$A=$ intervalo real pontual

$a_{1}=$ extremo inferior de $A$

$a_{2}=$ extremo superior $\operatorname{de} A$

$A^{(k)}=$ elemento de uma sequência de intervalos reais

$a_{1}^{(k)}=$ extremo inferior de $A^{(k)}$

$a_{2}^{(k)}=$ extremo superior $\operatorname{de} A^{(k)}$

$\mathcal{A}=$ matriz real

$a_{i j}=$ elemento de uma matriz real

$[A]=$ matriz intervalar real

$[A]=$ matriz intervalar real pontual

$A_{i j}=$ elemento de uma matriz intervalar real

$a_{i j 1}=$ extremo inferior de $A_{i j}$

$a_{i j 2}=$ extremo superior de $A_{i j}$

$\left[A^{(k)}\right]=$ elemento de uma sequência de matrizes intervalares reais

$A_{i j}^{(k)}=$ elemento de uma matriz $\left[A^{(k)}\right]$

$[x]=$ vetor intervalar real

$[x]=$ vetor intervalar real pontual

$X_{k}=$ elemento de um vetor intervalar real

$\left[x^{(k)}\right]=$ elemento de uma sequência de vetores intervalares reais 


\section{Introdução}

Consideremos o problema de auto-valores de matrizes

$$
\mathcal{A} x=\lambda x
$$

onde $\mathcal{A}$ é matriz real $n \times n, \lambda$, escalar e $x$, um vetor real não nulo de ordem $n$.

O objetivo desta dissertação é estudar e analisar métodos intervalares (métodos que utilizam a teoria da Matemática Intervalar) que limitam os auto-valores e respectivos auto-vetores de matrizes reais; também construir e analisar versões intervalares de conhecidos métodos iterativos para determinação dos auto-valores de matrizes e de métodos que determinam os auto-valores de $A$ através do cálculo dos zeros de seu polinômio característico.

Para isso, dividimos o trabalho em 05 capitulos:

No capítulo 1 - Matemática Intervalar - fazemos algumas consideraçōes gerais e um breve relato histórico sobre a Matemática Intervalar, e introduzimos os conceitos básicos necessários à compreensão e desenvolvimento da Dissertação, como: a noçâo de número intervalar, a aritmética intervalar na sua forma natural e arredondada, as propriedades métricas dos intervalos e o conceito de avaliação intervalar de uma função.

No capítulo 2 - Métodos Intervalares para limitar os autovalores e auto-vetores de matrizes simétricas - apresentamos, e analisamos criticamente, métodos intervalares já existentes para a solução do problema autovalores de matrizes simétricas.

No capítulo 3 - Versão Intervalar de Métodos para Determinação do Auto-valores através do Polinômio Característico da Matriz - desenvolvemos a versão intervalar de métodos numéricos para construção do polinômio característico de matrizes reais, e sugerimos alguns métodos intervalares para determinar os zeros desse polinômio. 
No capitulo 4 - Versão Intervalar de Métodos Iterativos para a Determinação dos Auto-valores de Matrizes - construímos versões intervalares dos métodos iterativos LR, de Rutishauser e QR, de Francis para a solução do problema de auto-valores de matrizes.

No capitulo 5 - Conclusões - aqui apresentamos as conclusões e observaçōes finais.

Por último, nas Referências Bibliográficas relacionamos os livros e artigos por nós consultados. 


\section{Capítulo 1}

\section{Matemática Intervalar}

Neste capítulo faremos algumas consideraçôes, falaremos um pouco da história da Matemática Intervalar e introduzimos os conceitos, as técnicas básicas e as propriedades, necessários à compreensão dos capitulos posteriores, e que podem ser encontrados em Alefeld [1].

\subsection{Um Breve Histórico}

Ao executarmos um processo numérico para a resoluçâo de um problema em um computador, onde os cálculos são efetuados com precisão finita, é necessário fazermos estudos para verificar se o resultado obtido está próximo da soluçào exata e saber qual foi o erro cometido.

A Análise Intervalar consegue resolver, ao mesmo tempo e automaticamente, fazendo uso de sub-rotinas adequadas, os vários erros comuns aos cálculos computacionais, tais como, erros de arredondamento da máquina, erros de truncamento de processos infinitos, dados iniciais calculados de forma imprecisa, propagação destes erros, etc...

A base da Matemática Intervalar é o intervalo, uma extensão de número real, representado por um par ordenado de números reais, seus extremos.

Os métodos numéricos intervalares, utilizam a aritmética intervalar e sub-rotinas para controlar os erros de arredondamento da máquina, obtendo-se desta forma, intervalos cujos extremos têm precisão finita e que contêm os resultados exatos de precisão infinita. Os objetivos da Matemática Intervalar, neste 
caso, tornam-se: produzir algoritmos computacionais para encontrar intervalos contendo as soluçōes procuradas e tornar estes intervalos tão pequenos quanto possível.

O uso de intervalos para controlar os erros de cálculos computacionais na Análise Numérica é recente, mas a idéia, de se obter um intervalo que contenha o resultado desejado é bem antiga. Temos o exemplo do "Método de Arquimedes" para aproximação do número $\pi$. Arquimedes considerou um círculo de diâmetro $d$, e deduziu um método para se calcular a razâo do comprimento da circunferência pelo diâmetro da seguinte forma: inicialmente considerou polígonos regulares, de 6 lados, inscritos e circunscritos à cicunferência e, a cada passo, dobrava o número de lados e calculava seus perímetros. Assim, obteve uma sequência crescente de limites inferiores e uma sequência decrescente de limites superiores para a razão do comprimento da circunferência pelo diâmetro, dividindo os perímetros dos polígonos inscritos e circunscritos pelo diâmetro.

Temos muitos outros exemplos de uso de intervalos na matemática, como a definição dos números reais através de intervalos encaixantes com extremos racionais, intervalos de confiança em Estatística, etc ...

A introdução do conceito de número intervalar na Análise Numérica, é devida a P. S. Dwyer que, em 1951, no livro "Linear Computations", define as operações aritméticas básicas para intervalos (adição, subtração, multiplicação, divisão) e é considerado o criador da Matemática Intervalar.

Desde a publicação do livro "Interval Analysis" de R. E. Moore da Universidade de Wisconsin, em 1966, e traduzido para o alemão em 1969, a Matemática Intervalar vem se desenvolvendo rapidamente. Em 1974, G. Alefeld (Universidade de Karlsruhe) e J. Herzberger. (Universidade de Oldenburg) publicaram o livro "Einführung in die Intervallrechnung" traduzido para o inglês em 1983. Em 1979, R. E. Moore publica o livro "Methods and Applications of Interval Analysis", que possui, em seu apêndice, uma vasta bibliografia sobre o assunto (760 títulos), incluindo trabalhos publicados e não publicados, cobrindo o período de 1951 a 1979 .

O periódico Freiburger Intervall-Berichte, publicado pela Universidade de Freiburg, Alemanha, sob coordenação do Professor K. L. E. Nickel divulga, desde 1978, tudo o que de mais significativo se produz, em todo o mundo, sobre Matemática Intervalar.

Inicialmente, este ramo da Análise Numérica, era conhecido como Análise Intervalar. Em 1974, L. Fox, da Universidade de Oxford, Inglaterra propôs o nome "Matemática Intervalar" para unificar as áreas como: análise 
intervalar, aritmética intervalar, topologia intervalar, etc ...

Vários simpósios internacionais sobre Matemática Intervalar foram realizados. O primeiro foi organizado por L. Fox, 1968, em Oxford, Inglaterra. Os trabalhos apresentados neste simpósio foram reunidos e editados por $\mathrm{E}$. $\mathrm{R}$. Hansen, em "Topics in Interval Analysis", Oxford Press, 1969. O segundo, realizado em majo de 1975, em Karsruhe, Alemanha, teve seus trabalhos editados por K. Nickel, em "Interval Mathematics", Lectures Notes in Computer Science, v.29, 1975. Em Freiburg, foram realizados simpósios internacionais em maio de 1980 e setembro de 1985 e seus trabalhos foram editados também por K. Nickel em "Interval Mathematics 1980", Academic Press, e "Interval Mathematics 1985", Lectures Notes in Computer Science, v.212, respectivamente.

\section{$1.2 \quad$ Número Intervalar}

Seja $\Re$ o conjunto dos números reais, cujos elementos serão denotados por letras minúsculas $(a, b, c, \ldots)$.

Definição 1.1 Define-se por número intervalar real ou intervalo real ou simplesmente intervalo o subconjunto de $\Re$ :

$$
\left[a_{1}, a_{2}\right]=\left\{a \in \Re: a_{1} \leq a \leq a_{2}\right\} .
$$

Denotaremos por $I(\Re)$ o conjunto de todos os intervalos reais, e os seus elementos por letras maiúsculas $(A, B, C, \ldots)$.

Definição 1.2 Seja $A=\left[a_{1}, a_{2}\right]$ intervalo real. Se $a_{1}=a_{2}$ entâo $A$ é chamado intervalo pontual ou degenerado.

Pode-se escrever qualquer elemento de $\Re$ como um elemento de $I(\Re)$, ou seja, $a \in \Re$, pode ser representado por $[a, a] \in I(\Re)$.

Definição 1.3 Sejam $A=\left[a_{1}, a_{2}\right]$ e $B=\left[b_{1}, b_{2}\right]$ intervalos reais.

- Se $t \in \Re$ étal que $a_{1} \leq t \leq a_{2}$ então dizemos que $t$ pertence a $A$, ou $s \in j a, t \in A$.

- $A$ e $B$ são ditos iguais se, e somente se, $a_{1}=b_{1}$ e $a_{2}=b_{2}$. 
- Dizemos que $A$ está contido em $B$ se, e somente se, $b_{1} \leq a_{1} e$ $a_{2} \leq b_{2}$, ou seja, $A \subset B \Leftrightarrow b_{1} \leq a_{1}$ e $a_{2} \leq b_{2}$.

- Dizemos que $A$ é menor do que $B$ se, e somente se, $a_{2}<b_{1}$, definindo assim uma ordem parcial para elementos de $I(\Re)$.

Definição 1.4 Sejam $A=\left[a_{1}, a_{2}\right]$ e $B=\left[b_{1}, b_{2}\right]$ intervalos reais.

- $A$ interseç̧ão de $A$ e $B, A \cap B$, é definida como o intervalo

$$
A \cap B=\left[\max \left\{a_{1}, b_{1}\right\}, \min \left\{a_{2}, b_{2}\right\}\right]
$$

Se $a_{1}>b_{2}$ ou $b_{1}>a_{2}$ então a intersecção é vazia, isto é, $A \cap B=\emptyset$.

- Se $A \cap B \neq \emptyset$, define-se a união de $A$ e $B, A \cup B$, como o intervalo

$$
A \cup B=\left[\min \left\{a_{1}, b_{1}\right\}, \max \left\{a_{2}, b_{2}\right\}\right]
$$

\subsection{Aritmética Intervalar}

Definição 1.5 Seja * um dos símbolos $\{+,-, \cdot, /\}$, representando uma das operaçôes aritméticas. As operações aritméticas sobre intervalos são definidas por

$$
A * B=\{a * b: a \in A, b \in B\}, \text { onde } A, B \in I(\Re),
$$

com a condição de que $0 \notin B$, para a divisão.

Sejam $A=\left[a_{1}, a_{2}\right]$ e $B=\left[b_{1}, b_{2}\right] \in I(\Re)$. É fácil mostrar que :

1. $A+B=\left[a_{1}+b_{1}, a_{2}+b_{2}\right]$.

2. $-B=\left[-b_{2},-b_{1}\right]$.

3. $A-B=A+(-B)=\left[a_{1}-b_{2}, a_{2}-b_{1}\right]$.

4. $A \cdot B=\left[\min \left\{a_{1} b_{1}, a_{1} b_{2}, a_{2} b_{1}, a_{2} b_{2}\right\}, \max \left\{a_{1} b_{1}, a_{1} b_{2}, a_{2} b_{1}, a_{2} b_{2}\right\}\right]$.

5. Se $0 \notin B, 1 / B=\left[1 / b_{2}, 1 / b_{1}\right]$.

6. Se $0 \notin B, A / B=A \cdot 1 / B=A \cdot\left[1 / b_{2}, 1 / b_{1}\right]$. 


\section{Propriedades da Aritmética Intervalar}

Propriedades 1.1 Sejam $A, B e C \in I(\Re)$, então valem:

1. para a adição intervalar

- $A+B=B+A$ (comutativa)

- $(A+B)+C=A+(B+C)$ (associativa)

- $\exists[0,0] \in I(\Re)$ tal que $A+[0,0]=[0,0]+A=A$ (elemento neutro)

- Se $A=\left[a_{1}, a_{2}\right]$ é tal que $a_{1} \neq a_{2}$ então $0 \in(A-A)$

Obs: nâo existe elemento inverso para a adição intervalar.

2. para a multiplicaçâo intervalar

- $A \cdot B=B \cdot A$ (comutativa)

- $(A \cdot B) \cdot C=A \cdot(B \cdot C)$ (associativa)

- $\exists[1,1] \in I(\Re)$ tal que $A \cdot[1,1]=[1,1] \cdot A=A$ (elemento neutro)

- Se $0 \notin A$ então $1 \in(A / A)$ Obs: não existe elemento inverso para a multiplicação intervalar.

3. - $A \cdot(B+C) \subseteq A \cdot B+A \cdot C$ (subdistributividade)

- Se $t \in \Re$, então $t \cdot(A+B)=t \cdot A+t \cdot B$

- $A \cdot(B+C)=A \cdot B+A \cdot C$, se $b \cdot c \geq 0$ para todo $b \in B$ e $c \in C$.

Prova: Ver Alefeld [1], capítulo 1.

Teorema 1.1 (Monotonicidade da Inclusão) Sejam $A_{1}, A_{2}, B_{1}$ e $B_{2} \in$ $I(\Re)$ tais que

$$
A_{1} \subseteq B_{1} \quad e \quad A_{2} \subseteq B_{2} .
$$

Entâo,para as operaçôes aritméticas intervalares $* \in\{+,-, \cdot, /\}$ segue que

$$
A_{1} * A_{2} \subseteq B_{1} * B_{2} \text {. }
$$

Prova:

$A_{1} \subseteq B_{1} \Rightarrow\left(a \in A_{1} \Rightarrow a \in B_{1}\right)$,

$A_{2} \subseteq B_{2} \Rightarrow\left(a \in A_{2} \Rightarrow a \in B_{2}\right)$,

então

$A_{1} * A_{2}=\left\{a_{1} * a_{2}: a_{1} \in A_{1}, a_{2} \in A_{2}\right\} \subseteq\left\{a_{1} * a_{2}: a_{1} \in B_{1}, a_{2} \in B_{2}\right\}=B_{1} * B_{2}$. 
Definição 1.6 Se $r(x)$ é uma operação unitária contínua sobre $\Re$, então

$$
r(X)=\left[\min _{x \in X} r(X), \max _{x \in X} r(X)\right]
$$

define uma operação unitária sobre $I(\Re)$.

Ex: $X^{k}(k \in \Re), \quad e^{X}, \quad \ln (X), \quad \operatorname{sen}(X), \quad \cos (X), \quad$ etc...

Note que se $0 \in X, X^{2} \neq X \cdot X$

Ex: $X=[-2,3]$

$X^{2}=[0,9]$

$X \cdot X=[-6,9]$

Definição 1.7 Sejam $A=\left[a_{1}, a_{2}\right]$ e $B=\left[b_{1}, b_{2}\right]$ intervalos reais. Define-se:

- Ponto médio de $A$ como o número real

$$
m(A)=\frac{a_{1}+a_{2}}{2}
$$

- Distância entre $A$ e $B$ como o número real

$$
d(A, B)=\max \left\{\left|a_{1}-b_{1}\right|,\left|a_{2}-b_{2}\right|\right\}
$$

- Valor absoluto de $A$ como o número real

$$
|A|=\max \left\{\left|a_{1}\right|,\left|a_{2}\right|\right\}=\max _{a \in A}|a|=d(A,[0,0])
$$

- Diâmetro de A como o número real

$$
D(A)=a_{2}-a_{1} \geq 0
$$

Propriedades 1.2 Sejam $A=\left[a_{1}, a_{2}\right], B=\left[b_{1}, b_{2}\right], C=\left[c_{1}, c_{2}\right]$ e $E=\left[e_{1}, e_{2}\right]$ intervalos reais. Então:

1. (a) $d(A, B) \geq 0 \quad$ e, $\quad d(A, B)=0 \Leftrightarrow A=B$

(b) $d(A, B)=d(B, A)$

(c) $d(A, C)+d(C, B) \geq d(A, B)$ 
(d) $d(A+B, A+C)=d(B, C)$

(c) $d(A+B, C+E) \leq d(A, C)+d(B, E)$

(f) $d(t \cdot A, t \cdot B)=|t| \cdot d(A, B), \quad t \in \Re$

(g) $d(A \cdot B, A \cdot C) \leq|A| \cdot d(B, C)$.

2. (a) $|A| \geq 0$ e, $|A|=0 \Leftrightarrow A=[0,0]$

(b) $|A+B| \leq|A|+|B|$

(c) $|t \cdot A|=|t| \cdot|A|, \quad t \in \Re$

(d) $|A \cdot B|=|A| \cdot|B|$

(e) $\operatorname{Se} A \subseteq B$ então $|A| \leq|B|$.

3. (a) $D(A)=|A-A|$

(b) $A \subseteq B \Rightarrow D(A) \leq D(B)$

(c) $D(A \pm B)=D(A)+D(B)$

(d) $D(A \cdot B) \leq D(A) \cdot|B|+|A| \cdot D(B)$

(e) $D(t \cdot A)=|t| \cdot D(A), \quad t \in \Re$

\section{Provas:}

Daremos as provas de algumas propriedades; as demais provas podem ser encontradas em Alefeld [1].

1.(a) $d(A, B)=\max \left\{\left|a_{1}-b_{1}\right|,\left|a_{2}-b_{2}\right|\right\} \geq 0 \mathrm{e}$, $d(A, B)=0 \Leftrightarrow \max \left\{\left|a_{1}-b_{1}\right|,\left|a_{2}-b_{2}\right|\right\}=0 \Leftrightarrow\left|a_{1}-b_{1}\right|=\left|a_{2}-b_{2}\right|=0 \Leftrightarrow$ $\left\{\begin{array}{l}\left|a_{1}-b_{1}\right|=0 \\ \left|a_{2}-b_{2}\right|=0\end{array} \Leftrightarrow\left\{\begin{array}{l}a_{1}-b_{1}=0 \\ a_{2}-b_{2}=0\end{array} \Leftrightarrow\left\{\begin{array}{l}a_{1}=b_{1} \\ a_{2}=b_{2}\end{array} \Leftrightarrow A=B\right.\right.\right.$.

1.(b) $d(A, B)=\max \left\{\left|a_{1}-b_{1}\right|,\left|a_{2}-b_{2}\right|\right\}=\max \left\{\left|b_{1}-a_{1}\right|,\left|b_{2}-a_{2}\right|\right\}$ $=d(B, A)$.

1.(c) $d(A, C)+d(C, B)=\max \left\{\left|a_{1}-c_{1}\right|,\left|a_{2}-c_{2}\right|\right\}+\max \left\{\left|c_{1}-b_{1}\right|,\left|c_{2}-b_{2}\right|\right\}$ $\geq \max \left\{\left|a_{1}-c_{1}\right|+\left|c_{1}-b_{1}\right|,\left|a_{2}-c_{2}\right|+\left|c_{2}-b_{2}\right|\right\}$ $\geq \max \left\{\left|a_{1}-c_{1}+c_{1}-b_{1}\right|,\left|a_{2}-c_{2}+c_{2}-b_{2}\right|\right\}$ $=\max \left\{\left|a_{1}-b_{1}\right|,\left|a_{2}-b_{2}\right|\right\}$ $=d(A, B)$

1.(d) $d(A+B, A+C)=d\left(\left[a_{1}+b_{1}, a_{2}+b_{2}\right],\left[a_{1}+c_{1}, a_{2}+c_{2}\right]\right)$ 


$$
\begin{aligned}
& =\max \left\{\left|\left(a_{1}+b_{1}\right)-\left(a_{1}+c_{1}\right)\right|,\left|\left(a_{2}+b_{2}\right)-\left(a_{2}+c_{2}\right)\right|\right\} \\
& =\max \left\{\left|b_{1}-c_{1}\right|,\left|b_{2}-c_{2}\right|\right\} \\
& =d(B, C)
\end{aligned}
$$

1.(e) $d(A+B, C+E)=d\left(\left[a_{1}+b_{1}, a_{2}+b_{2}\right],\left[c_{1}+e_{1}, c_{2}+e_{2}\right]\right)$

$$
\begin{aligned}
& =\max \left\{\left|\left(a_{1}+b_{1}\right)-\left(c_{1}+e_{1}\right)\right|,\left|\left(a_{2}+b_{2}\right)-\left(c_{2}+e_{2}\right)\right|\right\} \\
& =\max \left\{\left|\left(a_{1}-c_{1}\right)+\left(b_{1}-e_{1}\right)\right|,\left|\left(a_{2}-c_{2}\right)+\left(b_{2}-e_{2}\right)\right|\right\} \\
& \leq \max \left\{\left|\left(a_{1}-c_{1}\right)\right|+\left|\left(b_{1}-e_{1}\right)\right|,\left|\left(a_{2}-c_{2}\right)\right|+\left|\left(b_{2}-e_{2}\right)\right|\right\} \\
& \leq \max \left\{\left|a_{1}-c_{1}\right|,\left|a_{2}-c_{2}\right|\right\}+\max \left\{\left|b_{2}-e_{1}\right|,\left|b_{2}-e_{2}\right|\right\} \\
& =d(A, C)+d(B, E)
\end{aligned}
$$

1.(f) $d(t \cdot A, t \cdot B)=d\left(t \cdot\left[a_{1}, a_{2}\right], t \cdot\left[b_{1}, b_{2}\right]\right)$

Se $t \geq 0 \Rightarrow t \cdot\left[a_{1}, a_{2}\right]=\left[t \cdot a_{1}, t \cdot a_{2}\right]$

$$
t \cdot\left[b_{1}, b_{2}\right]=\left[t \cdot b_{1}, t \cdot b_{2}\right]
$$

\section{então}

$$
\begin{aligned}
d(t \cdot A, t \cdot B) & =d\left(\left[t \cdot a_{1}, t \cdot a_{2}\right],\left[t \cdot b_{1}, t \cdot b_{2}\right]\right) \\
& =\max \left\{\left|t \cdot a_{1}-t \cdot b_{1}\right|, \mid t \cdot a_{2}-t \cdot b_{2}\right\} \\
& =\max \left\{|t| \cdot\left|a_{1}-b_{1}\right|,|t| \cdot\left|a_{2}-b_{2}\right|\right\} \\
& =|t| \cdot \max \left\{\left|a_{1}-b_{1}\right|,\left|a_{2}-b_{2}\right|\right\} \\
& =|t| \cdot d(A, B)
\end{aligned}
$$

Se $t<0 \Rightarrow t \cdot\left[a_{1}, a_{2}\right]=\left[t \cdot a_{2}, t \cdot a_{1}\right]$

entào

$$
t \cdot\left[b_{1}, b_{2}\right]=\left[t \cdot b_{2}, t \cdot b_{1}\right]
$$

$$
\begin{aligned}
d(t \cdot A . t \cdot B) & =d\left(\left[t \cdot a_{2}, t \cdot a_{1}\right],\left[t \cdot b_{2}, t \cdot b_{1}\right]\right) \\
& =\max \left\{\left|t \cdot a_{2}-t \cdot b_{2}\right|, \mid t \cdot a_{1}-t \cdot b_{1}\right\} \\
& =\max \left\{|t| \cdot\left|a_{2}-b_{2}\right|,|t| \cdot\left|a_{1}-b_{1}\right|\right\} \\
& =|t| \cdot \max \left\{\left|a_{2}-b_{2}\right|,\left|a_{1}-b_{1}\right|\right\} \\
& =|t| \cdot d(A, B)
\end{aligned}
$$

$$
\text { 2.(d) } \begin{aligned}
|A \cdot B| & =\max _{x \in A \cdot B}|x|=\max _{a \in A, b \in B}|a \cdot b| \\
& =\max _{a \in A, b \in B}|a| \cdot|b|=\max _{a \in A}|a| \cdot \max _{b \in B}|b| \\
& =|A| \cdot|B|
\end{aligned}
$$

2.( $\epsilon)$ Seja $A \subseteq B$. Então, para todo $a \in A \Rightarrow a \in B$.

Assim. $|A|=\max _{a \in A}|a| \leq \max _{a \in B}|a|=|B|$. 


$$
\text { 3.(a) } \begin{aligned}
|A-A| & =\left|\left[a_{1}, a_{2}\right]-\left[a_{1}, a_{2}\right]\right|=\left|\left[a_{1}-a_{2}, a_{2}-a_{1}\right]\right| \\
& =\max \left\{\left|a_{1}-a_{2}\right|,\left|a_{2}-a_{1}\right|\right\}=\max \left\{\left|a_{2}-a_{1}\right|,\left|a_{2}-a_{1}\right|\right\} \\
& =\left|a_{2}-a_{1}\right|=a_{2}-a_{1} \\
& =D(A) .
\end{aligned}
$$

3.(c) $D(A+B)=D\left(\left[a_{1}, a_{2}\right]+\left[b_{1}, b_{2}\right]\right)=D\left(\left[a_{1}+b_{1}, a_{2}+b_{2}\right]\right)$

$$
\begin{aligned}
& =\left(a_{2}+b_{2}\right)-\left(a_{1}+b_{1}\right)=\left(a_{2}-a_{1}\right)+\left(b_{2}-b_{1}\right) \\
& =D(A)+D(B)
\end{aligned}
$$$$
D(A-B)=D\left(\left[a_{1}, a_{2}\right]-\left[b_{1}, b_{2}\right]\right)=D\left(\left[a_{1}-b_{2}, a_{2}-b_{1}\right]\right)
$$$$
=\left(a_{2}-b_{1}\right)-\left(a_{1}-b_{2}\right)=\left(a_{2}-a_{1}\right)+\left(b_{2}-b_{1}\right)
$$$$
=D(A)+D(B)
$$

\subsection{Vetores e Matrizes Intervalares}

Definição 1.8 - Um vetor intervalar real é um vetor cujos elementos são intervalos reais.

- Uma matriz intervalar real é uma matriz cujos elementos são intervalos reais.

Denotaremos por $V_{n}(I(\Re))$ o conjunto de todos os vetores intervalares reais de dimensão $n$ e seus elementos por letras minúsculas entre colchetes $([a],[b], \ldots)$. E por $M_{m n}(I(\Re))$ o conjunto de todas as matrizes intervalares reais de dimensão $m \times n$ e seus elementos por letras maiúsculas entre colchetes $([A],[B], \ldots)$.

Definição 1.9 Sejam as matrizes intervalares $[A]=\left(A_{i j}\right),[B]=\left(B_{i j}\right)$ $\in M_{m \pi}(I(\Re))$,

$$
[A]=[B] \text { se, e somente se, } A_{i j}=B_{i j} \text {, para } 1 \leq i \leq m, 1 \leq j \leq n \text {. }
$$

$[A] \subseteq[B]$ se $A_{i j} \subseteq B_{i j}$, para $1 \leq i \leq m, 1 \leq j \leq n$.

Definição 1.10 Seja a matriz $[A]=\left(A_{i j}\right) \in M_{m n}(I(\Re))$; se os elementos de $[A]$ sâo intervalos pontuais, $[A]$ é chamada matriz pontual e será denotada por $[A]$.

Definição 1.11 Sejam $[A]=\left(A_{i j}\right)$ e $[B]=\left(B_{i j}\right)$ matrizes intervalares reais. $A$ interseç̧ão de $[A]$ e $[B]$ é definida como a matriz intervalar

$$
[A] \cap[B]=\left(A_{i j} \cap B_{i j}\right), \quad 1 \leq i \leq m, \quad 1 \leq j \leq n
$$


Definição 1.12 Sejam as matrizes $[A]=\left(A_{i j}\right),[B]=\left(B_{i j}\right) \in M_{m n}(I(\Re))$. Definimos, adiçâo e subtração sobre $M_{m n}(I(\Re))$ por:

$$
\begin{aligned}
& {[A]+[B]=\left((A+B)_{i j}\right)=\left(A_{i j}+B_{i j}\right), \quad 1 \leq i \leq m, \quad 1 \leq j \leq n} \\
& {[A]-[B]=\left((A-B)_{i j}\right)=\left(A_{i j}-B_{i j}\right), \quad 1 \leq i \leq m, \quad 1 \leq j \leq n}
\end{aligned}
$$

Definição 1.13 Sejam as matrizes $[A]=\left(A_{i k}\right) \in M_{m p}(I(\Re))$ e $[B]=\left(B_{k j}\right) \in$ $M_{p n}(I(\Re))$. Define-se multiplicação intervalar de $[A]$ por $[B]$ como a matriz intervalar:

$$
[A] \cdot[B]=\left(\sum_{k=1}^{p} A_{i k} \cdot B_{k j}\right), \quad 1 \leq i \leq m, \quad 1 \leq j \leq n
$$

Definição 1.14 Seja a matriz $[A]=\left(A_{i j}\right) \in M_{n n}(I(\Re))$. Define-se por traço de $[A]$, o intervalo real :

$$
\operatorname{tr}([A])=\sum_{i=1}^{n} A_{i i}
$$

Definição 1.15 Sejam a matriz $[A]=\left(A_{i j}\right) \in M_{m n}(I(\Re))$ e o intervalo real $X$. Define-se multiplicação intervalar de $[A]$ por $X$ como a matriz intervalar:

$$
[A] \cdot X=X \cdot[A]=\left(X \cdot A_{i j}\right), \quad 1 \leq i \leq m, \quad 1 \leq j \leq n
$$

Dada a matriz intervalar real $[A]$ de ordem $n$, entende-se por matriz inversa intervalar de $[A]$ a matriz que contém as matrizes inversas de todas as matrizes pontuais pertencentes a $[A]$. Notação: $\left[A^{-1}\right]$.

O produto da matriz intervalar pela sua inversa intervalar contém a matriz identidade real, isto é:

$$
\begin{aligned}
& {[A] \cdot\left[A^{-1}\right] \supseteq\left[I_{n}\right]} \\
& {\left[A^{-1}\right] \cdot[A] \supseteq\left[I_{n}\right]}
\end{aligned}
$$

Entende-se por matriz ortogonal intervalar, a matriz intervalar $[Q]$ que satisfaz:

$$
\left[Q^{t}\right]=\left[Q^{-1}\right]
$$


Propriedades 1.3 Para matrizes e vetores intervalares valem as seguintes propriedades:

- $[A] \pm[B]=\{[A] \pm[B]:[A] \in[A],[B] \in[B]\}$, onde $[A]$ e $[B] \in M_{m n}(I(\Re))$.

- $\{[A] \cdot[B]:[A] \in[A],[B] \in[B]\} \subseteq\{[M]:[M] \in[A] \cdot[B]\}$, onde $[A] \in$ $M_{m n}(I(\Re)),[B] \in M_{n p}(I(\Re))$.

Propriedades 1.4 Se $[A],[B]$ e $[C]$, são matrizes intervalares reais, então valem:

- $[A]+[B]=[B]+[A]$

- $([A]+[B])+[C]=[A]+([B]+[C])$

- $[A]+[0]=[0]+[A]=[A]$, onde $[0]$ é a matriz nula

- $[A] \cdot[I]=[I] \cdot[A]=[A]$, onde $[I]$ é a matriz identidade real

- $([A]+[B]) \cdot[C] \subseteq[A] \cdot[C]+[B] \cdot[C]$

- $[A] \cdot([B]+[C]) \subseteq[A] \cdot[B]+[A] \cdot[C]$

(subdistributividade)

Provas: Ver Alefeld [1], capítulo 10.

Teorema 1.2 (Monotonicidade da Inclusão) Sejam $\left[A_{1}\right],\left[A_{2}\right],\left[B_{1}\right] \in\left[B_{2}\right]$ matrizes intervalares reais e $X, Y \in I(\Re)$, tais que

$$
\left[A_{1}\right] \subseteq\left[B_{1}\right], \quad\left[A_{2}\right] \subseteq\left[B_{2}\right] \quad \text { e } X \subseteq Y .
$$

Então, para $* \in\{+,-, \cdot\}$ temos

$$
\begin{gathered}
{\left[A_{1}\right] *\left[A_{2}\right] \subseteq\left[B_{1}\right] *\left[B_{2}\right] \quad e} \\
X \cdot\left[A_{1}\right] \subseteq Y \cdot\left[B_{1}\right] .
\end{gathered}
$$

Prova análoga à do Teorema 1.1. 
Definição 1.16 Sejam $[A]=\left(A_{i j}\right) e[B]=\left(B_{i j}\right) \in M_{m n}(I(\Re))$. Definimos:

- Matriz ponto médio de $[A]$ como a matriz real

$$
[M]([A])=\left(m\left(A_{i j}\right)\right)
$$

- Distância entre $[A]$ e $[B]$ como a matriz real não negativa $d([A],[B])=\left(d\left(A_{i j}, B_{i j}\right)\right), \quad 1 \leq i \leq m, \quad 1 \leq j \leq n ;$

- Valor absoluto de $[A]$ como a matriz real náo negativa

$$
\|[A] \mid=\left(\left|A_{i j}\right|\right), \quad 1 \leq i \leq m, \quad 1 \leq j \leq n ;
$$

- Diâmetro de $[A]$ como a matriz real não negativa

$$
D([A])=\left(D\left(A_{i j}\right)\right), \quad 1 \leq i \leq m, \quad 1 \leq j \leq n .
$$

Propriedades 1.5 Se $[A],[B],[C]$ e $[D]$ são matrizes intervalares reais e $[0]$ matriz nula intervalar, entâo valem:

- $d([A],[B])=[0] \Leftrightarrow[A]=[B],[A],[B] \in M_{m n}(I(\Re))$;

- $d([A]+[B],[A]+[C])=d([B],[C]),[A],[B],[C] \in M_{m n}(I(\Re))$;

- $d([A]+[B],[C]+[D]) \leq d([A],[C])+d([B],[D]),[A],[B],[C],[D]$ $\in M_{m n}(I(\Re))$;

- $d([A] \cdot[B],[A] \cdot[C]) \leq|[A]| \cdot d([B],[C]),[A] \in M_{m n}(I(\Re)),[B],[C] \in$ $M_{n p}(I(\Re))$;

- $d([A],[B]) \leq d([A],[C])+d([B],[C]),[A],[B],[C] \in M_{m n}(I(\Re))$;

- $|[A]| \geq[0] \quad$ e $|[A]|=[0] \Leftrightarrow[A]=[0],[A] \in M_{m n}(I(\Re))$;

- $[A] \subseteq[B] \Rightarrow|[A]| \leq|[B]|,[A],[B] \in M_{m n}(I(\Re))$;

- $|[A]+[B]| \leq|[A]|+|[B]|,[A],[B] \in M_{m n}(I(\Re))$;

- $|\boldsymbol{t} \cdot[A]|=|[A] \cdot t|=|t| \cdot|[A]|, t \in \Re,[A] \in M_{m n}(I(\Re))$; 
- $|[A] \cdot[B]| \leq|[A]| \cdot|[B]|,[A] \in M_{m n}(I(\Re)),[B] \in M_{n p}(I(\Re))$;

- $[A] \subseteq[B] \Rightarrow D([A]) \leq D([B]),[A],[B] \in M_{m n}(I(\Re))$;

- $D([A] \pm[B])=D([A])+D([B]),[A],[B] \in M_{m n}(I(\Re))$

- $D([A] \cdot[B]) \geq|[A]| \cdot D([B]),[A] \in M_{m n}(I(\Re)),[B] \in M_{n p}(I(\Re))$;

- $D([A] \cdot[B]) \geq D([A]) \cdot|[B]|,[A] \in M_{m n}(I(\Re)),[B] \in M_{n p}(I(\Re))$;

- $D(t \cdot[A])=|t| \cdot D([A]), t \in \Re,[A] \in M_{m n}(I(\Re))$.

Provas análogas às das Propriedades 1.2.

\subsection{Espaço Métrico $I(\Re)$}

Com a definição 1.7 de distância entre dois intervalos, a função

$$
\begin{gathered}
d: I(\Re) \times I(\Re) \longrightarrow \Re^{+} \\
d\left(\left[a_{1}, a_{2}\right],\left[b_{1}, b_{2}\right]\right)=\max \left\{\left|a_{1}-b_{1}\right|,\left|a_{2}-b_{2}\right|\right\}
\end{gathered}
$$

é uma métrica sobre $I(\Re)$ e $(I(\Re), d)$ é então um espaço métrico, que chamaremos de espaço métrico $I(\Re)$, pois para quaisquer $A, B, e C \in I(\Re)$ valem:

i) $d(A, B)=0 \Leftrightarrow A=B$

ii) $d(A, B)=d(B, A)$

iii) $d(A, B) \leq d(A, C)+d(C, B)$

(Ver Propriedades 1.2).

A distância $d$ entre dois intervalos $A, B \in I(\Re)$ reduz-se a distância entre reais quando tomamos intervalos pontuais. A métrica $d$ sobre $I(\Re)$ é uma generalização da distância entre dois pontos do espaço $\Re$.

vergência.

Podemos, agora, introduzir os conceitos de continuidade e de con- 


\section{Funçōes no $I(\Re)$}

Seja $(M, \rho), M \subseteq \Re$ um espaço métrico com a função distância $\rho$.

Seja o espaço métrico $I(\Re)$ com a distância $d$.

Seja $F: M \longrightarrow I(\Re)$ uma função com valores intervalares.

Definição 1.17 A função intervalar $F$ é contínua em $x \in M$, se, para todo $\epsilon>0$ existe um $\delta>0$ tal que, para todo $y \in M$, com $\rho(x, y)<\delta$, então $d(F(x), F(y))<\epsilon$.

Entendemos por função real racional, uma função nas variáveis reais $x_{1}, \ldots, x_{n}$, cuja expressão funcional $f\left(x_{1}, \ldots, x_{n}\right)$ é composta de um número finito de operandos reais e operaçôes aritméticas reais. E por função intervalar racional, uma função nas variáveis intervalares $X_{1}, \ldots, X_{n}$, cuja expressão funcional $F\left(X_{1}, \ldots, X_{n}\right)$ é composta de um número finito de operandos intervalares e operações aritméticas intervalares.

Vamos considerar, a partir de agora, $f$ função real, contínua e definida em algum subconjunto $M$ de $\Re^{n}$,

$$
f: M \longrightarrow \Re^{n}
$$

Definição 1.18 Seja $f\left(x_{1}, \ldots, x_{n}\right)$ uma função real racional. A função intervalar racional $F\left(X_{1}, \ldots, X_{n}\right)$ é uma extensão ou avaliação intervalar de $f$, st $F\left(x_{1}, \ldots, x_{n}\right)=f\left(x_{1}, \ldots, x_{n}\right)$ para $x_{i} \in X_{i}$, argumentos reais.

Definição 1.19 A extensão intervalar natural de uma função real racional $f, \dot{e}$ a funçâo em que substituímos as variáveis reais por intervalares $\epsilon$ as operaçôes aritméticas reais por operações aritméticas intervalares.

Pretendemos limitar a imagem de uma função real em algum intervalo (ou conjunto de intervalos) através de extensão intervalar.

Se $f\left(x_{1}, \ldots, x_{n}\right)$ é uma função racional real, onde cada variável aparece somente uma vez na expressão, então a avaliaçâo intervalar correpondente, $F\left(X_{1}, \ldots, X_{n}\right)$, fornecerá a imagem de $f$, para $x_{i} \in X_{i}, i=1,2, \ldots, n$, isto é,

$$
F\left(X_{1}, \ldots, X_{n}\right)=\left\{f\left(x_{1}, \ldots, x_{n}\right): x_{i} \in X_{i}, i=1,2, \ldots, n\right\} .
$$


Por isso, para uma função racional real de uma variável $x$, onde esta variável aparece $n$ vezes em sua expressâo funcional, é interessante substituir cada ocorrência de $x$ por outras variáveis $x_{1}, \ldots, x_{n}$.

Se a função é um polinômio $p(x)=a_{0}+a_{1} x+\cdots+a_{n-1} x^{n-1}+a_{n} x^{n}$, seja $a_{0}+x\left(a_{1}+\cdots+x\left(a_{n-1}+x a_{n}\right) \cdots\right)$ a forma de Horner para o polinômio. Devido à propriedade da subdistributividade dos intervalos temos que:

$A_{0}+A_{1} X+A_{2} X X+A_{3} X X X+\cdots+A_{n} X X \cdots X \supseteq$ $A_{0}+X\left(A_{1}+X\left(A_{2}+\cdots+X\left(A_{n}\right) \cdots\right)\right)$.

Conclui-se que a avaliação intervalar da expressão do polinômio na forma de Horner produz intervalo de diâmetro menor que a avaliação intervalar da expressão original.

Ex: Seja o polinômio real $p(x)=3-4 x+\frac{1}{2} x^{2}$;

queremos saber, por exemplo, os valores de $p(x)$ para $x \in[1,2]$.

A extensão intervalar natural de $p(x)$ é o polinômio intervalar $P(X)=[3,3]-[4,4] X+\left[\frac{1}{2}, \frac{1}{2}\right] X^{2}$.

$P([1,2])=[3,3]-[4,4][1,2]+\left[\frac{1}{2}, \frac{1}{2}\right][1,2]^{2}=[-4.5,1]$.

Usando a propriedade da subdistributividade, encontramos a seguinte extensão intervalar para $p(x)$ :

$Q(X)=[3,3]+X\left(-[4,4]+X\left[\frac{1}{2}, \frac{1}{2}\right]\right)$.

$Q([1,2])=[3,3]+[1,2]\left(-[4,4]+[1,2]\left[\frac{1}{2}, \frac{1}{2}\right]\right)=[-4,0]$.

Vemos que $P([1,2]) \supset Q([1,2])$.

Deve-se tomar cuidado quando a avaliação intervalar for feita para um intervalo que contenha o 0 , pois se $0 \in X, X^{2} \neq X \cdot X$.

Se $P(X)=A_{0}+A_{1} X+A_{2} X X+A_{3} X X X+\cdots+A_{n} X X \cdots X$ e $R(X)=A_{0}+A_{1} X+A_{2} X^{2}+A_{3} X^{3}+\cdots+A_{n} X^{n}$, então para $0 \in X$, $P(X) \neq R(X)$.

\section{Convergência}

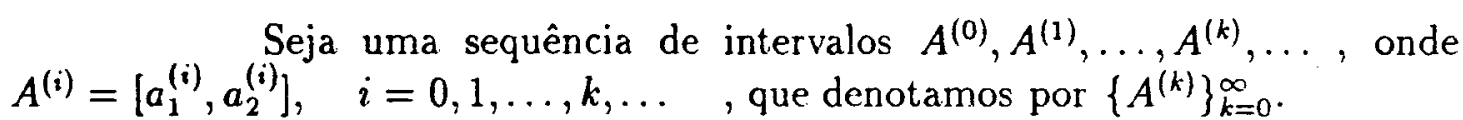


Definiçāo 1.20 Uma sequência de intervalos $\left\{A^{(k)}\right\}_{k=0}^{\infty}$ converge para um intervalo $A=\left[a_{1}, a_{2}\right]$ se, e somente se, as sequências dos extremos inferiores $\left\{a_{1}^{(k)}\right\}_{k=0}^{\infty}$ $e$ dos extremos superiones $\left\{a_{2}^{(k)}\right\}_{k=0}^{\infty}$ convergem para $a_{1}$ e $a_{2}$ respectivamente, isto $\dot{e}$,

$$
\lim _{k \rightarrow \infty} A^{(k)}=A \Leftrightarrow\left(\begin{array}{ll}
\lim _{k \rightarrow \infty} a_{1}^{(k)}=a_{1} & e \\
\lim _{k \rightarrow \infty} a_{2}^{(k)}=a_{2} &
\end{array}\right)
$$

ou ainda

$$
\lim _{k \rightarrow \infty} A^{(k)}=A \Leftrightarrow \lim _{k \rightarrow \infty} d\left(A^{(k)}, A\right)=0 \Leftrightarrow\left(\begin{array}{ll}
\lim _{k \rightarrow \infty}\left|a_{1}^{(k)}-a_{1}\right|=0 & e \\
\lim _{k \rightarrow \infty}\left|a_{2}^{(k)}-a_{2}\right|=0 &
\end{array}\right)
$$

Definição 1.21 Uma sequência $\left\{A^{(k)}\right\}_{k=0}^{\infty}$ tal que $A^{(0)} \supseteq A^{(1)} \supseteq \ldots \supseteq A^{(k)}, \ldots e ́$ dita uma sequência de intervalos encaixantes.

Teorema 1.3 Uma sequência de intervalos encaixantes $\left\{A^{(k)}\right\}_{k=0}^{\infty}$ converge para o intervalo $A=\bigcap_{k=0}^{\infty} A^{(k)}$.

\section{Prova:}

Seja $A^{(k)}=\left[a_{1}^{(k)}, a_{2}^{(k)}\right]$. Então,

$\left\{a_{1}^{(k)}\right\}$ é uma sequência de números reais não decrescente limitada por $a_{2}^{(k)}$, e seja $a_{1}$ o seu limite e,

$\left\{a_{2}^{(k)}\right\}$ é uma sequência de números reais não crescente limitada por $a_{1}^{(k)}$ : e seja $a_{2}$ o seu limite.

Como $a_{1}^{(k)} \leq a_{2}^{(k)}$ para todo $k$, temos que $a_{1} \leq a_{2}$.

Logo $\left\{A^{(k)}\right\}_{k=0}^{\infty}$ converge para $\left[a_{1}, a_{2}\right]=A=\bigcap_{k=0}^{\infty} A^{(k)}$.

Corolário 1.3.1 Toda sequência de matrizes intervalares $m \times n,\left\{\left[A^{(k)}\right]\right\}_{k=0}^{\infty}$, tal que $\left[A^{(0)}\right] \supseteq\left[A^{(1)}\right] \supseteq \ldots \supseteq\left[A^{(k)}\right], \ldots$, converge para uma matriz intervalar $[A]=\left(A_{i j}\right)$, onde $A_{i j}=\bigcap_{k=0}^{\infty} A_{i j}^{(k)}, \quad 1 \leq i \leq m, \quad 1 \leq j \leq n$.

Definição 1.22 Uma sequência de intervalos $\left\{A^{(k)}\right\}_{k=0}^{\infty}$ tem convergência finita se existe um número inteiro positivo $m$ tal que $A^{(k)}=A^{(m)}$ para todo $k \geq m$. Esta sequência será chamada de sequência convergente em $m$ passos. 
Exemplo:

$$
\begin{aligned}
& A^{(0)}=[0.000,1.000] \\
& A^{(1)}=[0.333,0.667] \\
& A^{(2)}=[0.444,0.556] \\
& A^{(3)}=[0.481,0.519] \\
& A^{(4)}=[0.493,0.507] \\
& A^{(5)}=[0.497,0.503] \\
& A^{(6)}=[0.499,0.501] \\
& A^{(7)}=[0.499,0.501]
\end{aligned}
$$

Observação: Usando a representação de máquina com precisão finita, qualquer sequência de intervalos encaixantes tem convergência finita.

Teorema 1.4 Seja $\left\{A^{(k)}\right\}_{k=0}^{\infty}$ tal que existe um número real $t, t \in A^{(k)}$, $k=0,1,2, \ldots$ Definimos a sequência de intervalos $\left\{B^{(k)}\right\}_{k=0}^{\infty}$ por:

$$
\begin{gathered}
B^{(0)}=A^{(0)} \\
B^{(k+1)}=A^{(k+1)} \cap B^{(k)}, \quad k=0,1,2, \ldots
\end{gathered}
$$

Então $\left\{B^{(k)}\right\}_{k=0}^{\infty}$ é uma sequência de intervalos encaixantes com limite $B e$

$$
t \in B \subseteq B^{(k)}, \quad k=0,1,2, \ldots
$$

\subsection{Aritmética Intervalar Arredondada}

Na prática, a aritmética real de precisão infinita não é possível de ser executada e sempre estaremos limitados a aritmética aproximada de precisão finita da máquina, o que provoca erros de arredondamento.

A aritmética intervalar, por si só, nào nos dará os limites superiores e inferiores para a solução exata de muitos problemas. Em toda operação aritmética intervalar executada por um computador, usa-se a aritmética real para se obter os extremos dos intervalos resultantes e, portanto, continuamos com os erros de arredondamento da máquina. Surge, então, a necessidade de criarmos sub-rotinas que arredondem o extremo inferior, para a esquerda, e o extremo superior, para a direita, de modo a se obter um intervalo de máquina que contenha o intervalo verdadeiro.

Sabemos que um computador pode representar somente um conjunto finito de números que, em aritmética de ponto flutuante, escreve-se sob a 
forma:

$$
\pm 0, d_{1} d_{2} \ldots d_{n} \cdot b^{e}= \pm m \cdot b^{e}
$$

onde

$1 \leq d_{1} \leq b-1$ e $0 \leq d_{i} \leq b-1, i=2, \ldots, n$, b é a base, geralmente igual a $2, e \in Z$ é o expoente, limitado por $e_{\min } \leq e \leq e_{\max }$, dependendo da máquina a ser utilizada ( $Z$ é o conjunto dos números inteiros).

Denotemos por $\Re_{M}$ o conjunto de todos os números de máquina. $\hat{E}$ fácil ver que $\Re_{M} \subset \Re$.

Os números reais podem ser arredondados pelos números de máquina utilizando-se a função definida a seguir.

Definição 1.23 Seja $f_{a}$ uma função definida da seguinte forma:

$$
\begin{gathered}
f_{a}: \Re \longrightarrow \Re_{M} \\
f_{a}(x)=\bar{x}=m \cdot b^{e}
\end{gathered}
$$

$f_{a}$ é chamada funçâo arredondamento se

$$
\forall x, y \in \Re, x \leq y \Rightarrow f_{a}(x) \leq f_{a}(y) .
$$

Definição 1.24 Seja $\uparrow: \Re \longrightarrow \Re_{M}$ uma função arredondamento, $\uparrow$ é chamada de função arredondamento direcionado para cima (por excesso) se

$$
\forall x \in \Re, x \leq \uparrow(x)
$$

Definição 1.25 Seja $\downarrow: \Re \longrightarrow \Re_{M}$ uma função arredondamento, $\downarrow$ é chamada $d \epsilon$ função arredondamento direcionado para baixo (por falta) se

$$
\forall x \in \Re, \downarrow(x) \leq x
$$

Escolhemos as funções arredondamento $\uparrow \mathrm{e} \downarrow$ de tal forma que:

$$
\uparrow(x)=-(\downarrow(-x)), \quad x \in \Re .
$$

e para qualquer função arredondamento $f_{a}(x)$,

$$
\uparrow(x) \leq f_{a}(x) \quad \text { e } \quad \downarrow(x) \geq f_{a}(x), \quad x \in \Re^{+}
$$


Utilizamos estas funções arredondamento em $\Re$ para arredondar convenientemente os extremos dos intervalos de $I(\Re)$ obtidos por alguma operação aritmética intervalar.

Denotaremos por $I\left(\Re_{M}\right)$ o conjunto dos intervalos cujos extremos estão em $\Re_{M}$, ou seja, $I\left(\Re_{M}\right)=\left\{\left[x_{1}, x_{2}\right]: x_{1}, x_{2} \in \Re_{M}\right\} \subset I(\Re)$, ou ainda, $I\left(\Re_{M}\right)$ é o conjunto dos intervalos de máquina.

Definição 1.26 Seja $₫$ uma função, da seguinte forma:

$$
\begin{gathered}
I: I(\Re) \longrightarrow I\left(\Re_{M}\right) \\
X \longmapsto I(X)
\end{gathered}
$$

Ié chamada função arredondamento intervalar se

i) $X \in I(\Re) \Rightarrow X \subseteq 1(X)$

ii) $X, Y \in I(\Re), X \subseteq Y \Rightarrow I(X) \subseteq\rfloor(Y)$

Propriedades 1.6 $X \in I\left(\Re_{M}\right) \Rightarrow \downarrow(X)=X$

Se $X=\left[x_{1}, x_{2}\right]$, então escolhemos as funções arredondamento $\uparrow, \downarrow$ e $\$ de tal forma que:

$$
\begin{gathered}
I(X)=\llbracket\left(\left[x_{1}, x_{2}\right]\right)=\left[\downarrow\left(x_{1}\right), \uparrow\left(x_{2}\right)\right] \\
\text { ou } I(X)=I\left(\left[x_{1}, x_{2}\right]\right)=\left\lfloor\downarrow\left(x_{1}\right),-\left(\downarrow\left(-x_{2}\right)\right)\right]
\end{gathered}
$$

O nosso próximo passo será associar toda esta discussão sobre arredondamento às operações aritméticas intervalares. A isto chamaremos de Aritmética Intervalar Arredondada (AIA).

Teorema 1.5 Sejam as operações aritméticas intervalares e $A_{1}, A_{2}, B_{1}$ e $B_{2}$ $\in I\left(\Re_{M^{\prime}}\right)$ tais que $A_{1} \subseteq B_{1}$ e $A_{2} \subseteq B_{2}$. Se $C_{1}=\rrbracket\left(A_{1} * A_{2}\right)$ e $C_{2}=I\left(B_{1} * B_{2}\right)$ então $C_{1} \subseteq C_{2}$.

Teorema 1.6 Sejam $A, B \in I\left(\Re_{M}\right), * \in\{+,-, \cdot, /\}$ operações aritméticas intervalares $\epsilon$ seja $\$ a função arredondamento intervalar. Então temos que

$$
A * B \subseteq \mathfrak{l}(A * B) \in I\left(\Re_{M}\right) .
$$


Prova:

Seja o intervalo $C=A * B$; pela definição $1.26 C \subseteq\rfloor(C)$.

Portanto $A * B \subseteq\rfloor(A * B)$.

Este teorema garante que o intervalo resultante calculado pela AIA contém o intervalo verdadeiro (de precisão infinita). Estes resultados calculados numericamente satisfazem ao Teorema da Monotonicidade da Inclusão.

A AlA elimina a acumulação de erros de arredondamento de cálculos e permite controlar a propagação dos erros nos dados iniciais.

\section{Implementação da Aritmética Intervalar Arredondada}

As definiçôes 1.5 e 1.26 dão a estrutura para a realização da implementação da AIA. A implementação da primeira definição em qualquer linguagem de programação de alto nível é simples, por isso não nos preocuparemos com esta parte. A implementação dos arredondamentos convenientes, entretanto, requer algum trabalho, pois não estão usualmente disponiveis em linguagens de programação.

Faremos a implementação da função arredondamento direcionado para baixo $(\downarrow)$ no procedimento LOW, em linguagem PASCAL. Da equação 1.2 vemos que não há necessidade de se implementar a função $\uparrow$.

Um intervalo $A=\left[a_{1}, a_{2}\right]$ é definido como um ARRAY [1..2] OF $\mathrm{REAL}$, onde A[1] conterá o valor $a_{1}$ e A[2] o valor $a_{2}$.

A parte principal do procedimento dependerá das seguintes propriedades das operações aritméticas da máquina:

1. Tipos de arredondamento usados quando operamos os números reais em ponto flutuante:

(a) truncamento dos dígitos, em excesso no comprimento da palavra, para o número de máquina menor mais próximo ou,

(b) arredondamento para o número em ponto flutuante mais próximo.

2. Comportamento nas vizinhanças do 0 . Se o resultado da operação real é, em valor absoluto, menor do que o menor número de máquina positivo, então: 
(a) igualamos o resultado a 0 , se quizermos arredondar o extremo inferior ou,

(b) fazemos o resultado igual ao menor número de máquina, em valor absoluto, com o sinal do resultado real, se quizermos arredondar o extremo superior.

3. Quando houver "overflow", uma extensão sensível das operações intervalares de máquina é somente possível em casos expecionais. Geralmente interrompemos os cálculos quando isto acontece.

Sendo $k$ o número de dígitos da mantissa $m$ da representação 1.1 ,

$\operatorname{minpos}:=\min \left\{x: x \in \Re_{M}, x>0\right\}$

fator $\mathbf{A}:=1-b^{1-k}$, fator de conversão usado para o caso do item 1.(a),

fator $B:=1-\frac{1}{2} b^{1-k}$, fator de conversão usado para o caso do item 1.(b),

são as constantes que, dependendo da máquina usada, serão utilizadas nos arredondamentos convenientes.

Como a máquina que estamos usando arredonda os valores de acordo com o item 1.(b), o procedimento será da forma:

function LOW(var $x$ : real) : real;

begin

if $x \geq 0$

then begin

$x:=x *$ fator $\mathrm{B}$

if $x<=$ minpos then $x:=0$

end

else begin

$x:=x /$ fator $\mathrm{B}$

if $x>-$ minpos then $x:=-$ minpos

end

LOW: $:=x$

end; 
Para exemplificar o uso da function LOW, daremos agora o procedimento da adição intervalar:

interval: array [1..2] of real;

procedure ADIÇÃO (a,b : interval; var $\mathbf{c}:$ interval);

var soma :real;

begin

$$
\begin{aligned}
& \text { soma:=a[1]+b[1]; } \\
& \text { c[1]:= LOW }(\text { soma }) \\
& \text { soma: }=\mathrm{a}[2]+\mathrm{b}[2] \\
& \text { c[2]:= -LOW(-soma); }
\end{aligned}
$$

end; 


\section{Capítulo 2}

\section{Métodos Intervalares para Limitar os Auto-valores e Auto-vetores de Matrizes Simétricas}

Apresentaremos neste capítulo, um método intervalar para limitar os auto-vetores de matrizes reais simétricas devido a Nickel [12], e uma considerável modificação deste método devida a Zhiying [17], que o torna iterativo e limitador também dos auto-valores da matriz.

\subsection{Método Proposto por Nickel}

Inicialmente apresentaremos o método real que motivou o método intervalar para a limitaçào dos auto-vetores de matrizes simétricas.

Seja $\mathcal{A}=\left(a_{i j}\right)$ uma matriz real simétrica de ordem $n$. Seja $\lambda$ um auto-valor de $\mathcal{A}$. Aplicando o método de eliminação de Gauss à matriz singular 
$\mathcal{A}-\lambda I_{n}$, após convenientes trocas de linhas e colunas, obtemos a matriz:

$$
\mathcal{B}=\left(\begin{array}{ccccccc}
b_{11} & b_{12} & \ldots & b_{1 t} & b_{1 t+1} & \ldots & b_{1 n} \\
& b_{22} & \ldots & b_{2 t} & b_{2 t+1} & \ldots & b_{2 n} \\
& & \ddots & \vdots & \vdots & & \vdots \\
& & & b_{t t} & b_{t t+1} & \ldots & b_{t n} \\
& & & & 0 & \ldots & 0 \\
& & & & \vdots & & \vdots \\
& & & & 0 & \ldots & 0
\end{array}\right)
$$

com $b_{i i} \neq 0$, para $i=1,2, \ldots, t$ e $r=n-t$ linhas nulas.

Escolhendo $r$ vetores, $r \times 1$, linearmente independentes $y_{1}, y_{2}, \ldots$, $y_{r}$, define-se $x_{i}=\left(\begin{array}{c}z_{i} \\ y_{i}\end{array}\right)$, onde $z_{i}$, para $i=1,2, \cdots, r$ são vetores, $t \times 1$, desconhecidos.

Resolve-se, então, para cada $x_{i}, i=1,2, \cdots, r$, o sistema linear $\mathcal{B} x_{i}=0$, de $t$ equações.

Pode-se mostrar facilmente que o número $r$ de linhas nulas da matriz $\mathcal{B}$, é a multiplicidade do auto-valor $\lambda$ e que os $r$ vetores $x_{1}, x_{2}, \ldots, x_{r}$, são auto-vetores linearmente independentes correspondentes a $\lambda$.

Teoricamente o algoritmo acima é bem eficiente, porém, ao usarmos a aritmética de ponto flutuante de um computador, nem sempre conseguimos bons resultados, devido aos erros de arredondamento, que podem modificar o posto da matriz $\mathcal{B}=\mathcal{A}-\lambda I_{n}$, arbitrariamente.

Usando aritmética intervalar, Nickel apresentou a versão intervalar do método dado acima.

Analogamente ao caso real, seja $\mathcal{A}$, matriz real simétrica de ordem $n$ e seja $\Lambda=[\lambda, \bar{\lambda}]$ um intervalo real, tal que os auto-valores $\lambda_{1}, \lambda_{2}, \ldots, \lambda_{n}$ de $A$ satisfazem

$$
\left\{\begin{array}{l}
\lambda_{i} \in \Lambda, \text { para } i=1,2, \ldots, r \mathrm{e} \\
\lambda_{i} \notin \Lambda, \text { para } i=r+1, r+2, \ldots, n
\end{array}\right.
$$

Seja $[B]=[A]-\Lambda\left[I_{n}\right]$ matriz intervalar $n \times n$, onde $[A]=\left(A_{i j}\right)$ e $A_{i j}=\left[a_{i j}, a_{i j}\right]$. Aplicando o Método de Eliminação de Gauss Intervalar em $[B] \mathrm{e}$ 
após convenientes trocas de linhas e colunas, obtemos a matriz intervalar:

$$
[B]=\left(\begin{array}{ccccccc}
B_{11} & B_{12} & \ldots & B_{1 t} & B_{1 t+1} & \ldots & B_{1 n} \\
& B_{22} & \ldots & B_{2 t} & B_{2 t+1} & \ldots & B_{2 n} \\
& & \ddots & \vdots & \vdots & & \vdots \\
& & & B_{t t} & B_{t t+1} & \ldots & B_{t n} \\
& & & & B_{t+1 t+1} & \ldots & B_{t+1 n} \\
& & & & \vdots & & \vdots \\
& & & & B_{n t+1} & \ldots & B_{n n}
\end{array}\right)
$$

com $0 \notin B_{i i}$, para $i=1,2, \ldots, t$ e $0 \in B_{i k}$, para $i, k=t+1, \ldots, n$.

Sendo $m=n-t$, escolhem-se $m$ vetores intervalares pontuais, $m \times 1$, linearmente independentes $\left[y_{1}\right],\left[y_{2}\right], \ldots,\left[y_{m}\right]$, e define-se $\left[x_{i}\right]=\left(\begin{array}{l}{\left[z_{i}\right]} \\ {\left[y_{i}\right]}\end{array}\right)$, onde $\left[z_{i}\right]$, para $i=1,2, \cdots, m$ são vetores intervalares, $t \times 1$, desconhecidos.

Resolve-se, então, para cada $x_{i}, i=1,2, \cdots, m$, o sistema linear intervalar $[B]\left[x_{i}\right]=[0]$, de $t$ equações, onde $[0]$ é um vetor intervalar pontual, $n \times 1$, com todos os seus elementos iguais a $[0,0]$.

Teorema 2.1 Para o algoritmo intervalar acima valem as seguintes propriedades:

1) $m \geq r$

2) $\left\{x_{1}, x_{2}, \ldots, x_{r}\right\} \subseteq\left\{\left[x_{1}\right],\left[x_{2}\right], \ldots,\left[x_{m}\right]\right\}$

onde $x_{i}, i=1,2, \ldots, r$ são auto-vetores da matriz $\mathcal{A}$.

Prova:

Todos os valores finais e intermediários do algoritmo real são obtidos dos valores $a_{i j}$ da matriz $\mathcal{A}$ e de $\lambda$ e, no caso intervalar são obtidos de $a_{i j}$ e $\Lambda$. Como $\lambda \in \Lambda$, pela propriedade intervalar da monotonicidade da inclusão, o teorema fica provado.

Portanto, no caso intervalar, o número de linhas da matriz $[B]$ que contém o zero em todos os seus elementos, pode ser maior que o número de linhas nulas de $\mathcal{B}$ do caso real.

\subsection{Método Proposto por Zhiying}

Quando $m=r$ temos que $x_{i} \in\left[x_{i}\right], \quad i=1,2, \ldots, r$. Considerando somente o caso em que $m=r$ e a partir do método descrito acima, 
Zhiying construiu um método iterativo de modo a obter melhores resultados para limitar os auto-valores e correspondentes auto-vetores.

Dada uma matriz simétrica real $\mathcal{A}=\left(a_{i j}\right), n \times n$ e um intervalo real $\Lambda=[\lambda, \bar{\lambda}]$ que contém um único auto-valor de multiplicidade $r, \lambda$, de $\mathcal{A}$, novamente define-se a matriz intervalar $[B]=[A]-\Lambda\left[I_{n}\right]$ e obtém-se o vetor intervalar $\left[x_{i}\right], i=1,2, \ldots, r$ pelo método anterior. Podemos supor, sem perda de generalidade, que $0 \notin \Lambda$.

Temos, então, $\left[x_{i}\right]=\left(\begin{array}{l}{\left[z_{i}\right]} \\ {\left[y_{i}\right]}\end{array}\right), i=1,2, \ldots, r$, onde $\left[y_{i}\right]$ são vetores intervalares pontuais linearmente independentes, $r \times 1, \mathrm{e}$, portanto, pelo menos um elemento de cada $\left[y_{i}\right]$ é diferente de zero. Pode-se supor, sem perda de generalidade, que o último elemento dos vetores $\left[y_{i}\right]$ é igual a $[1,1]$, isto é, $\left[y_{i r}, y_{i r}\right]=[1,1]$, para $i=1,2, \ldots, r$.

Assim,

$$
\left[x_{i}\right]=\left(\begin{array}{c}
{\left[z_{i}\right]} \\
{\left[y_{i 1}, y_{i 1}\right]} \\
\vdots \\
{\left[y_{i r-1}, y_{i r-1}\right]} \\
{[1,1]}
\end{array}\right) \text {,para } i=1,2, \ldots, r
$$

De $x_{i} \in\left[x_{i}\right]$ tem-se que $x_{i n}=1$, para $i=1,2, \ldots, r$.

Como $\mathcal{A} x_{i}=\lambda x_{i}$, sua última equação se escreve como:

$$
\sum_{k=1}^{n} a_{n k} x_{i k}=\lambda x_{i n}=\lambda
$$

Mas, $x_{i} \in\left[x_{i}\right]$, então $x_{i k} \in X_{i k}$ e $A_{n k}=\left[a_{n k}, a_{n k}\right]$, pela equação 2.1 temos que

$$
\lambda \in \sum_{k=1}^{n} A_{n k} X_{i k}
$$

E como $\lambda \in \Lambda$, usando 2.2 concluímos que

$$
\lambda \in \sum_{k=1}^{n} A_{n k} X_{i k} \cap \Lambda, \quad \text { para } i=1,2, \ldots, r .
$$


Sabe-se que $0 \notin \Lambda$. Logo, $\lambda \neq 0$ e $\mathcal{A} x_{i}=\lambda x_{i} \Rightarrow x_{i}=\frac{\mathcal{A} x_{i}}{\lambda}, \quad i=1,2, \ldots, r$.

Portanto,

$$
x_{i} \in \frac{[A]\left[x_{i}\right]}{\Lambda} \text { e } \quad x_{i} \in \frac{[A]\left[x_{i}\right]}{\Lambda} \cap\left[x_{i}\right], \quad i=1,2, \ldots, r
$$

Com base na discussão acima, podemos agora, construir o seguinte algoritmo:

\section{Algoritmo Intervalar}

Dados:

$\mathcal{A}$ - matriz real simétrica de ordem $n$

$\Lambda$ - intervalo real, com $0 \notin \Lambda$ e contendo apenas o auto-valor $\lambda$ de $\mathcal{A}$ de multiplicidade $r$.

1. Definir a matriz intervalar

$$
[B]=[A]-\Lambda\left[I_{n}\right] .
$$

2. Aplicar o método de eliminação de Gauss Intervalar para resolver:

$$
[B]\left[x_{i}^{(0)}\right]=[0], i=1,2, \ldots, r,
$$

3. Definir $\Lambda^{(0)}=\Lambda$.

4. Para $k=1,2, \ldots$, executar os passos abaixo até que $\left[x_{i}^{(k+1)}\right]=\left[x_{i}^{(k)}\right]$.

(a) Para $i=1,2, \ldots, r$ :

$$
\begin{aligned}
& \text { calcular } \Lambda_{i}^{(k+1)}=\sum_{j=1}^{n} A_{n j} X_{i j}^{(k)} \cap \Lambda^{(k)} . \\
& \text { calcular }\left[x_{i}^{(k+1)}\right]=\frac{[A]\left[x_{i}^{(k)}\right]}{\Lambda^{(k)}} \cap\left[x_{i}^{(k)}\right] .
\end{aligned}
$$

(b) Calcular $\Lambda^{(k+1)}=\Lambda_{1}^{(k+1)} \cap \Lambda_{2}^{(k+1)} \cap \cdots \cap \Lambda_{r}^{(k+1)}$

5. Fim do algoritmo. 
Teorema 2.2 (Convergência) As sequências $\left\{\Lambda^{(k)}\right\}$ e $\left\{\left[x_{i}^{(k)}\right]\right\}$, geradas pelo algoritmo intervalar, são convergentes, isto é,

$$
\text { quando } k \rightarrow \infty,\left\{\begin{array}{l}
\Lambda^{(k)} \rightarrow \Lambda^{*}, \\
{\left[x_{i}^{(k)}\right] \rightarrow\left[x_{i}^{*}\right], \text { para } i=1,2, \ldots, r .}
\end{array}\right.
$$

Além disso, $\lambda \in \Lambda^{*}, x_{i} \in\left[x_{i}^{*}\right]$, para $i=1,2, \ldots, r$.

Prova:

A prova a seguir é dada por Zhiying [17].

Do algoritmo iterativo intervalar anterior, tem-se que:

$$
\begin{gathered}
\Lambda^{(0)} \supseteq \Lambda^{(1)} \supseteq \cdots \supseteq \Lambda^{(k)} \supseteq \cdots \\
{\left[x_{i}^{(0)}\right] \supseteq\left[x_{i}^{(1)}\right] \supseteq \cdots \supseteq\left[x_{i}^{(k)}\right] \supseteq \cdots, \text { para } i=1,2, \ldots, r .}
\end{gathered}
$$

$\mathrm{E} \lambda \in \Lambda^{(0)}, x_{i} \in\left[x_{i}^{(0)}\right]$

Daí, segue o Teorema.

\subsection{Observações}

Um dos problemas observados no algoritmo intervalar acima é que podemos obter $\left[x_{i}^{(k)}\right]=\left[x_{i}^{(k+1)}\right]$, porém, com o diâmetro de $\left[x_{i}^{(k)}\right]$ ainda não suficientemente pequeno. Para contornar isto, podemos dividir o intervalo $\Lambda^{(k)}$ ao meio, conforme sugestão de J. Zhiying [17], obtendo dois intervalos $\Lambda^{\prime}$ e $\Lambda^{\prime \prime}$ e tomarmos aquele em que posto $\left([A]-\Lambda\left[I_{n}\right]\right)=n-r$ e continuarmos o processo $\operatorname{com}\left[x_{i}^{(0)}\right]=\left[x_{i}^{(k)}\right]$. Se, em ambos os intervalos, o posto for igual a $n-r$, escolhe-se arbitrariamente um deles e abandona-se ao encontrarmos, por exemplo, $m=0$ ou alguma intersecção do algoritmo intervalar igual a vazio, e retoma-se o processo com o outro intervalo.

Pelo fato de reiniciarmos a iteração com $\left[x_{i}^{(0)}\right]=\left[x_{i}^{(k)}\right]$ ao fazermos a bissecção de $\Lambda^{(k)}$, os auto-vetores obtidos nem sempre têm boa precisâo. Através de uma pequena modificação no método dado, recalculando os auto-vetores iniciais $\left[x_{i}^{(0)}\right]$ usando o método da seção 2.1 cada vez que dividimos o intervalo $\Lambda^{(k)}$ ao meio, isto é, retornando ao passo 1 do algoritmo, conseguimos melhorar significativamente os auto-vetores.

$\mathrm{Na}$ tentativa de estendermos o algoritmo para matrizes quaisquer, verificamos que, se os auto-vetores correspondentes ao auto-valor $\lambda \in \Lambda$ forem linearmente independentes, o comportamento é o mesmo que para matrizes simétricas. 


\subsection{Exemplos}

1)

matriz real : $\left(\begin{array}{rrr}5 & -2 & -4 \\ -2 & 2 & 2 \\ -4 & 2 & 5\end{array}\right)$ e $\Lambda^{(0)}=[9,12]$

$m=1$,

para $k=202$,

$\Lambda^{(k)}=[9.9999999969 e+00,1.0000000004 e+01]$

$\left[x_{1}^{(k)}\right]=\left(\begin{array}{c}{[-1.0000000051 \mathrm{e}+00,-9.9999999689 \mathrm{e}-01]} \\ {[4.9999999880 \mathrm{e}-01,5.0000000184 \mathrm{e}-01]} \\ {[1.0000000000 \mathrm{e}+00,1.0000000000 \mathrm{e}+00]}\end{array}\right.$,

2)

matriz real : $\left(\begin{array}{lll}1 & -3 & 3 \\ 3 & -5 & 3 \\ 6 & -6 & 4\end{array}\right)$ e $\Lambda^{(0)}=[-3.0,-0.1]$

$m=2$,

para $k=358$,

$\Lambda^{(k)}=[-2.0000000038 e+00,-1.9999999944 e+00]$

$\left[x_{1}^{(k)}\right]=\left(\begin{array}{l}{[1.0000000073 \mathrm{e}+00,-9.9999999376 \mathrm{e}-01]} \\ {[0.0000000000 \mathrm{e}+00,0.0000000000 \mathrm{e}+00]} \\ {[1.0000000000 \mathrm{e}+00,1.0000000000 \mathrm{e}+00]}\end{array}\right)$

$\left[x_{2}^{(k)}\right]=\left(\begin{array}{c}{[-2.0000000146 \mathrm{e}+00,-1.9999999875 \mathrm{e}+00]} \\ {[-1.0000000000 \mathrm{e}+00,0.0000000000 \mathrm{e}+00]} \\ {[1.0000000000 \mathrm{e}+00,1.0000000000 \mathrm{e}+00]}\end{array}\right)$ 


\section{Capítulo 3}

\section{Versão Intervalar de Métodos para Determinação dos Auto-valores através do Polinômio Característico da Matriz}

Outra maneira bem conhecida de se determinar os auto-valores de uma matriz real, é calcular o seu polinômio característico, e encontrar os zeros desse polinômio. Com o objetivo de fazer a versão intervalar dessa técnica, tomamos o polinômio característico intervalar de uma matriz intervalar real e sugerimos alguns métodos intervalares para limitação de suas raízes.

3.1 Versāo Intervalar de Métodos Numéricos para Determinação do Polinômio Característico de Matrizes

Consideremos a matriz real $\mathcal{A}=\left(a_{i j}\right), n \times n, p(\lambda)=\operatorname{det}\left(\mathcal{A}-\lambda I_{n}\right)$ é o polinômio característico de $\mathcal{A}$.

$$
p(\lambda)=(-1)^{n}\left(p^{(0)} \lambda^{n}-p^{(1)} \lambda^{n-1}-\ldots-p^{(n-1)} \lambda-p^{(n)}\right)
$$

onde $p^{(0)}=1$. 
Substituindo a matriz real $\mathcal{A}$ pela matriz intervalar pontual $[A]$, onde $\left(A_{i j}\right)=\left[a_{i j}, a_{i j}\right], i, j=1, \ldots, n$, nosso objetjvo é encontrar os coeficientes do polinómio característico intervalar de $[A]$,

$$
P(\Lambda)=(-1)^{n}\left(P^{(0)} \Lambda^{n}-P^{(1)} \Lambda^{n-1}-\ldots-P^{(n-1)} \Lambda-P^{(n)}\right)
$$

onde $p^{(i)} \in P^{(i)}$ para $i=1, \ldots, n$ e $P^{(0)}=[1,1]$.

Para isso, estudamos as versões intervalares de métodos que determinam os coeficientes do polinômio característico de matrizes reais.

\subsubsection{Método de Leverrier-Faddeev}

Seja a matriz intervalar real pontual $[A]$, como definida acima. A versão intervalar do método de Leverrier-Faddeev é dada pela construção de uma sequência de matrizes intervalares $\left[A_{1}\right],\left[A_{2}\right], \ldots,\left[A_{n}\right]$, e uma sequência de intervalos reais $P^{(1)}, P^{(2)}, \ldots, P^{(n)}$ da seguinte forma:

$$
\begin{gathered}
{\left[A_{1}\right]=[A], \quad P^{(1)}=\operatorname{tr}\left(\left[A_{1}\right]\right), \quad\left[B_{1}\right]=\left[A_{1}\right]-P^{(1)}\left[I_{n}\right]} \\
{\left[A_{2}\right]=[A]\left[B_{1}\right], \quad P^{(2)}=\frac{\operatorname{tr}\left(\left[A_{2}\right]\right)}{[2,2]}, \quad\left[B_{2}\right]=\left[A_{2}\right]-P^{(2)}\left[I_{n}\right]} \\
{\left[A_{n-1}\right]=[A]\left[B_{n-2}\right], \quad P^{(n-1)}=\frac{\operatorname{tr}\left(\left[A_{n-1}\right]\right)}{[n-1, n-1]}, \quad\left[B_{n-1}\right]=\left[A_{n-1}\right]-P^{(n-1)}\left[I_{n}\right]} \\
{\left[A_{n}\right]=[A]\left[B_{n-1}\right], \quad P^{(n)}=\frac{\operatorname{tr}\left(\left[A_{n}\right]\right)}{[n, n]}, \quad\left[B_{n}\right]=\left[A_{n}\right]-P^{(n)}\left[I_{n}\right] .}
\end{gathered}
$$

Os intervalos reais $P^{(1)}, P^{(2)}, \ldots, P^{(n)}$ são os coeficientes intervalares do polinômio intervalar 3.2. Pelo Teorema da Monotonicidade da Inclusão temos que os intervalos resultantes $P^{(1)}, \ldots, P^{(n)}$ contêm os coeficientes reais $p^{(1)}, \ldots, p^{(n)}$ do polinômio característico 3.1 , respectivamente.

Quando a matriz $[A]$ é de ordem alta $(>6)$ e/ou os seus auto-valores são valores altos em módulo, os intervalos obtidos têm diâmetros grandes, isto é, 
conseguimos um intervalo que contém o respectivo coeficiente real mas com uma margem de erro muito grande.

Cremos que isto ocorre por causa das $(n-1)$ multiplicações de matrizes que existem no método, pois, notamos também que $D\left(P^{(1)}\right)<D\left(P^{(2)}\right)<\cdots<D\left(P^{(n)}\right)$, isto é, o diâmetro dos coeficientes intervalares cresce com a ordem da matriz.

\subsubsection{Método de Danilevsky}

Seja a matriz intervalar pontual $[A]$. A versão intervalar do método de Danilevsky por nós construída consiste em reduzir a matriz $[A]$ à forma:

$$
[P]=\left(\begin{array}{ccccc}
P^{(1)} & P^{(2)} & \ldots & P^{(n-1)} & P^{(n)} \\
{[1,1]} & {[0,0]} & \ldots & {[0,0]} & {[0,0]} \\
\ldots & \ldots & \ldots & \ldots & \ldots \\
{[0,0]} & {[0,0]} & \ldots & {[1,1]} & {[0,0]}
\end{array}\right)
$$

por meio de $(n-1)$ transformaçōes de similaridade, ou seja:

$$
[P]=\left[M_{1}^{-1}\right]\left[M_{2}^{-1}\right] \cdots\left[M_{n-1}^{-1}\right][A]\left[M_{n-1}\right] \cdots\left[M_{2}\right]\left[M_{1}\right]
$$

onde $\left[M_{i}^{-1}\right] \cdot\left[M_{i}\right] \supseteq\left[I_{n}\right] \quad$ para $i=1,2, \ldots, n-1, \mathrm{e}$

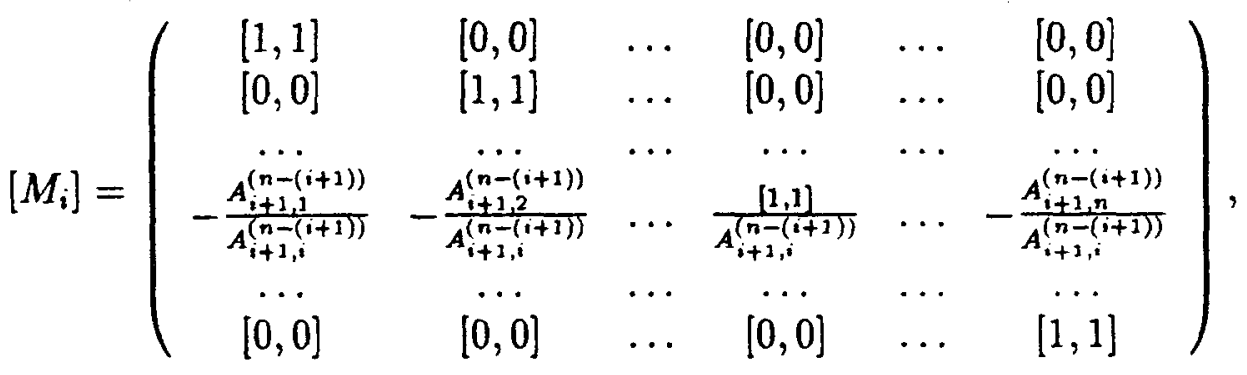

$$
\begin{aligned}
& {\left[M_{i}^{-1}\right]=\left(\begin{array}{cccccc}
{[1,1]} & {[0,0]} & \ldots & {[0,0]} & \ldots & {[0,0]} \\
{[0,0]} & {[1,1]} & \ldots & {[0,0]} & \ldots & {[0,0]} \\
\ldots & \ldots & \ldots & \ldots & \ldots & \ldots \\
A_{i+1,1}^{(n-(i+1))} & A_{i+1,2}^{(n-(i+1))} & \ldots & A_{i+1, i}^{(n-i+1))} & \ldots & A_{i+1, n}^{(n-i+1))} \\
\ldots & \ldots & \ldots & \ldots & \ldots & \ldots \\
{[0,0]} & {[0,0]} & \ldots & {[0,0]} & \ldots & {[1,1]}
\end{array}\right)}
\end{aligned}
$$


$\mathrm{e}\left[A^{(k)}\right]=\left(A_{i j}^{(k)}\right)$

$\left[A^{(0)}\right]=[A]$

$\left[A^{(k)}\right]=\left[\dot{M}_{n-k}^{-1}\right] \cdots\left[M_{n-1}^{-1}\right][A]\left[M_{n-1}\right] \cdots\left[M_{n-k}\right]$

Portanto, $[P]=\left[A^{(n-1)}\right]$.

Os elementos $P^{(i)}, i=1, \ldots, n$ da matriz $[P]$ são os coeficientes do polinômio característico 3.2 da matriz $[A]$ e contêm os respectivos coeficientes do polinômio característico $3.1 \mathrm{da}$ matriz real $\mathcal{A}$.

Para que todas as transformações acima sejam realizadas, vamos supor, sempre, que $A_{i+1, i}^{(n-(i+1))}$, não contenha o zero, para $i=1, \ldots, n-1$.

\subsection{Métodos Intervalares para Determinação de Zeros de Polinômios}

A seguir, apresentaremos métodos intervalares para determinação de zeros de funções, e que serão por nós utilizados, para determinar os zeros dos polinônios característicos obtidos pelos métodos acima. Estudamos o capítulo 7 de Moore [10] e os artigos de Herzberger [8], Hansen [7] e Arthur [2] para encontrar métodos intervalares mais eficientes e abrangentes para determinação de zeros de polinômios. Descreveremos os seguintes métodos:

\subsubsection{Método de Newton-Moore}

Moore [10] apresenta o seguinte método, conhecido por Método de Newton-Moore.

Considere $f(x)$ uma função real racional, tal que $f(x)$ é limitada em $x \in\left[x_{1}, x_{2}\right]$. Então $f$ tem uma derivada contínua, $f^{\prime}$, em $\left[x_{1}, x_{2}\right]$ e para $x, y \in\left[x_{1}, x_{2}\right]$ temos, do Teorema do Valor Médio

$$
f(x)=f(y)+f^{\prime}(y+\theta(x-y))(x-y) \text { para } \theta \in[0,1]
$$

Se $x$ é um zero de $f$ e $f^{\prime}$ não se anula em $\left[x_{1}, x_{2}\right]$, então:

$$
x=y-\frac{f(y)}{f^{\prime}(y+\theta(x-y))} \text {. }
$$

Seja $f(x)$ uma função real racional com apenas uma raiz simples em $\left[x_{1}, x_{2}\right]$. Seja $F^{\prime}(X)$ a extensão intervalar de $f^{\prime}$ tal que $0 \notin F^{\prime}(X)$. Define-se 
a função intervalar $N:\left[x_{1}, x_{2}\right] \longrightarrow I(\Re)$, por

$$
N(X)=m(X)-\frac{f(m(X))}{F^{\prime}(X)}, \quad X \subset\left[x_{1}, x_{2}\right] .
$$

Quando os coeficientes de $f(x)$ são conhecidos somente em certos intervalos, toma-se a extensão intervalar $F(X)$ de $f(x)$ e define-se $N(X)$ por:

$$
N(X)=m(X)-\frac{F(m(X))}{F^{\prime}(X)}, \quad X \subset\left[x_{1}, x_{2}\right]
$$

Para $X^{(0)}=\left[x_{1}^{(0)}, x_{2}^{(0)}\right]$, construímos a sequência de intervalos $X^{(1)}$, $X^{(2)}, \ldots$ do seguinte modo:

$$
X^{(i+1)}=N\left(X^{(i)}\right) \cap X^{(i)}, \quad i=0,1,2, \ldots
$$

Observação: $N(X)$ será definida somente quando $f$ possuir um zero simples em $X$.

Lema 3.1 Seja $N(X)$ definida como em 3.3. Se o intervalo $X$ contém apenas um zero, $x^{*}$, de $f$, então $x^{*} \in N(X)$.

Prova: Ver Moore [10].

Lema 3.2 Se $N(X) \cap X$ é vazio, então não existem zeros de $f(x)$ em $X$. Caso contrário, $N(X) \cap X$ contém pelo menos o zero de $f$ que está em $X$.

Prova: Ver Moore [10].

\subsubsection{Método de Herzberger}

Intervalar:

Herzberger [8] faz a seguinte modificação no Método de Newton

Se $f\left(x_{1}^{(0)}\right)<0$, consideramos o seguinte intervalo:

$$
Y^{(i)}=\left\{\begin{array}{lll}
{\left[x_{1}^{(i)}, m\left(X^{(i)}\right)\right],} & \text { se } & f\left(m\left(X^{(i)}\right)\right)>0 \\
{\left[m\left(X^{(i)}\right), x_{2}^{(i)}\right],} & \text { se } & f\left(m\left(X^{(i)}\right)\right)<0 .
\end{array}\right.
$$


Definimos

$$
X^{(i+1)}=\left\{m\left(X^{(i)}\right)-\frac{f\left(m\left(X^{(i)}\right)\right)}{F^{\prime}\left(Y^{(i)}\right)}\right\} \cap Y^{(i)}, \quad i=0,1,2, \ldots
$$

Analogamente, se $f\left(x_{1}^{(0)}\right)>0$ e, como $Y^{(i)} \subset X^{(i)}$, pelo Teorema da Monotonicidade da Inclusâo, $F^{\prime}\left(Y^{(i)}\right) \subset F^{\prime}\left(X^{(i)}\right)$ e portanto,

$$
\left\{m\left(X^{(i)}\right)-\frac{f\left(m\left(X^{(i)}\right)\right)}{F^{\prime}\left(Y^{(i)}\right)}\right\} \cap Y^{(i)} \subset\left\{m\left(X^{(i)}\right)-\frac{f\left(m\left(X^{(i)}\right)\right)}{F^{\prime}\left(X^{(i)}\right)}\right\} \cap X^{(i)}
$$

Daí concluímos que o método de Herzberger apresenta melhores resultados que o de Moore.

\subsubsection{Método de Hansen}

Em Hansen [7] encontramos outra considerável modificação do Método de Newton Intervalar, que passaremos a expor:

Considere a função $f(x)$ em que a variável $x$ ocorre mais de uma vez.

Como sugerido na seção 1.5 , substitui-se cada ocorrência da variável $x$ por outras variáveis $x_{1}, x_{2}, \ldots, x_{n}$ construindo a função $g\left(x_{1}, x_{2}, \ldots, x_{n}\right)$ tal que $g(x, x, \ldots, x)=f(x)$.

Considerando $g(x)$ como uma função de uma variável simples $x_{1}$, a expansâo de $g(x)$ em série nos fornece:

$$
g\left(x_{1}, x_{2}, \ldots, x_{n}\right)=g\left(y_{1}, x_{2}, \ldots, x_{n}\right)+\left(x_{1}-y_{1}\right) \frac{\partial}{\partial x_{1}} g\left(\epsilon_{1}, x_{2}, \ldots, x_{n}\right),
$$

$\epsilon_{1} \in\left[x_{1}, y_{1}\right]$

Agora, considerando $g\left(y_{1}, x_{2}, \ldots, x_{n}\right)$ como uma função de uma variável simples $x_{2}$, obtem-se:

$$
g\left(y_{1}, x_{2}, \ldots, x_{n}\right)=g\left(y_{1}, y_{2}, x_{3}, \ldots, x_{n}\right)+\left(x_{2}-y_{2}\right) \frac{\partial}{\partial x_{2}} g\left(y_{1}, \epsilon_{2}, x_{3}, \ldots, x_{n}\right),
$$

$\epsilon_{2} \in\left[x_{2}, y_{2}\right]$

E assim sucessivamente até que: 


$$
g\left(y_{1}, y_{2}, \ldots, y_{n-1}, x_{n}\right)=g\left(y_{1}, y_{2}, \ldots, y_{n}\right)+\left(x_{n}-y_{n}\right) \frac{\sim}{\partial x_{n}} g\left(y_{1}, y_{2}, \ldots, y_{n-1}, \epsilon_{n}\right)
$$

$\epsilon_{n} \in\left[x_{n}, y_{n}\right]$

Logo,

$g\left(x_{1}, x_{2}, \ldots, x_{n}\right)=g\left(y_{1}, y_{2}, \ldots, y_{n}\right)+\left(x_{1}-y_{1}\right) \frac{\partial}{\partial x_{1}} g\left(\epsilon_{1}, x_{2}, \ldots, x_{n}\right)+$

$\left(x_{2}-y_{2}\right) \frac{\partial}{\partial x_{2}} g\left(y_{1}, \epsilon_{2}, x_{3}, \ldots, x_{n}\right)+\ldots+\left(x_{n}-y_{n}\right) \frac{\partial}{\partial x_{n}} g\left(y_{1}, y_{2}, \ldots, y_{n-1}, \epsilon_{n}\right)$.

$g\left(x_{1}, x_{2}, \ldots, x_{n}\right)=g\left(y_{1}, y_{2}, \ldots, y_{n}\right)+\sum_{i=1}^{n}\left(x_{i}-y_{i}\right) \frac{\partial}{\partial x_{i}} g\left(y_{1}, \ldots, y_{i-1}, \epsilon_{i}, x_{i+1}, \ldots, x_{n}\right)$

onde $\epsilon_{i} \in\left[x_{i}, y_{i}\right], i=1, \ldots, n$.

Então:

$g\left(y_{1}, y_{2}, \ldots, y_{n}\right)=g\left(x_{1}, x_{2}, \ldots, x_{n}\right)+\sum_{i=1}^{n}\left(y_{i}-x_{i}\right) \frac{\partial}{\partial x_{i}} g\left(y_{1}, \ldots, y_{i-1}, \epsilon_{i}, x_{i+1}, \ldots, x_{n}\right)$.

tanto $\epsilon_{i} \in X_{i}$.

Tomamos intervalos iniciais $X_{1}, \ldots, X_{n}$, tais que $x_{i}, y_{i} \in X_{i}$, e por-

Seja $x_{1}=x_{2}=\ldots=x_{n}=x, y_{1}=y_{2}=\ldots=y_{n}=y$ e deixando $\epsilon_{i} \in X_{i i}$ para algum intervalo $X_{i i}$, temos

$$
g(y, y, \ldots, y)=g(x, x, \ldots, x)+\sum_{i=1}^{n}(y-x) \frac{\partial}{\partial x_{i}} g\left(X_{i 1}, \ldots, X_{i i-1}, X_{i i}, x, \ldots, x\right),
$$

ou seja,

$$
f(y)=f(x)+(y-x) \sum_{i=1}^{n} \frac{\partial}{\partial x_{i}} g\left(X_{i 1}, \ldots, X_{i i-1}, X_{i i}, x, \ldots, x\right)
$$

Supondo $y$ raiz de $f$, obtemos:

$$
y=x-\frac{f(x)}{\sum_{i=1}^{n} \frac{\partial}{\partial x_{i}} g\left(X_{i 1}, \ldots, X_{i i-1}, X_{i i}, x, \ldots, x\right)}
$$

Em nosso caso, cada um dos intervalos $X_{i 1}, X_{i 2}, \ldots, X_{i i}$ para $i=$ $1, \ldots, n$ são iguais a $X$. Usa-se notação diferente apenas para enfatizar que eles sào independentes. 
Assim, para $x \in X$, define-se

$$
N_{n}(X)=x-\frac{f(x)}{G^{\prime}(X)}
$$

onde

$$
\begin{gathered}
G^{\prime}(X)=\frac{\partial}{\partial x_{1}} g(X, x, \ldots, x)+\frac{\partial}{\partial x_{2}} g(X, X, x, \ldots, x)+\cdots \\
+\frac{\partial}{\partial x_{n}} g(X, X, \ldots, X) .
\end{gathered}
$$

sem perda de generalidade, faremos $x=m(X) \in X$

Substituindo-se $N\left(X^{(i)}\right)$ por $N_{n}\left(X^{(i)}\right)$ na equaçâo 3.5 , obtém-se a vantagem de que certos argumentos aparecem na forma real e, em geral fornecem resultados de diâmetro menor. $\mathrm{E} 0 \notin G^{\prime}(X)$ é menos restritivo do que $0 \notin F^{\prime}(X)$. é um polinômio.

Faremos, agora, algumas considerações para o caso em que a função

$$
\begin{aligned}
& \text { Seja } p(x)=a_{0}+a_{1} x+\cdots+a_{n-1} x^{n-1}+a_{n} x^{n} . \\
& \text { e } P(X)=a_{0}+a_{1} X+\cdots+a_{n-1} X^{n-1}+a_{n} X^{n} . \\
& P^{\prime}(X)=a_{1}+2 a_{2} X+\cdots+(n-1) a_{n-1} X^{n-2}+n a_{n} X^{n-1} .
\end{aligned}
$$

Reescrevendo o polinômio na forma de Horner; $p(x)=a_{0}+x\left(a_{1}+x\left(a_{2}+\cdots+x\left(a_{n}\right) \cdots\right)\right)$, e substituindo cada ocorrência da. variável $x$ por variáveis $x_{1}, x_{2} \ldots, x_{n}$, construímos a função:

$$
\begin{aligned}
g\left(x_{1}, \ldots, x_{n}\right) & =a_{0}+x_{1}\left(a_{1}+x_{2}\left(a_{2}+\cdots+x_{n}\left(a_{n}\right) \cdots\right)\right) \\
& =a_{0}+a_{1} x_{1}+a_{2} x_{1} x_{2}+\cdots+a_{n-1} x_{1} x_{2} \ldots x_{n-1}+a_{n} x_{1} x_{2} \ldots x_{n} .
\end{aligned}
$$

Calculando $G^{\prime}(X)$ por 3.6 temos que,

$$
\begin{aligned}
G^{\prime}(X)=a_{1}+a_{2} x & +a_{3} x x+\cdots \\
+a_{2} X+a_{3} X x+\cdots & +a_{n} X x \cdots x \\
& +a_{3} X X+\cdots+a_{n} X X \cdots x+\cdots \\
& +a_{n} X X \cdots X
\end{aligned}
$$




$$
G^{\prime}(X)=\sum_{j=1}^{n}[\left(a_{j}+\sum_{k=j+1}^{n} a_{k} x^{k-j}\right) \underbrace{X \cdot X \cdot \ldots \cdot X] .}_{(j-1) \text { vezes }}
$$

Como $x \in X$ e pela propriedade da subdistributividade, vê-se facilmente que $G^{\prime}(X) \subset P^{\prime}(X)$.

Sabemos que se $0 \in X, X^{2} \neq X \cdot X$. Ao fazermos substituição de variáveis em polinônios, alguns problemas podem ocorrer.

Por exemplo, se, $p(x)=x^{3}+4 x-16, \quad p^{\prime}(x)=3 x^{2}+4$ e $P^{\prime}(X)=3 X^{2}+4$

Seja $X=[-2,3]$, Então, $P^{\prime}([-2,3])=[4,31]$.

Se definirmos $g\left(x_{1}, x_{2}, x_{3}\right)=x_{1} x_{2} x_{3}+4 x_{1}-16$, obtemos

$G^{\prime}(X)=x^{2}+x X+X X+4$

então, se $x=1$

$G^{\prime}([-2,3])=[-3,17]$

logo $G^{\prime}(X) \not \subset P^{\prime}(X)$.

obtendo assim,

Para evitar este problema, quando $0 \in X$, tomamos $x=0$ em 3.7,

$$
G^{\prime}(X)=\sum_{k=1}^{n} a_{k} X^{k-1}
$$

logo, $G^{\prime}(X)=4+X^{2}=[4,13] \subset P^{\prime}(X)$.

Como $G^{\prime}(X) \subset P^{\prime}(X)$ temos:

$$
m(X)-\frac{p(m(X))}{G^{\prime}(X)} \subset m(X)-\frac{p(m(X))}{P^{\prime}(X)}
$$

obtendo melhores resultados que no método da Newton-Moore.

A grande "frustração" de todos os métodos que acabaram de ser apresentados é que se $x \in X^{(0)}$ (intervalo inicial) é uma raiz múltipla de $f(x)$ ou se existe mais de uma raiz de $f(x)$ em $X^{(0)}, N\left(X^{(0)}\right)$ não está definida. $O$ próximo método procura resolver estas dificuldades. 


\subsubsection{Método de Arthur}

Baseando-se no método de Derr, que é quadraticamente convergente,

$$
x^{(i+1)}=x^{(i)}-\frac{f^{(m-1)}\left(x^{(i)}\right)}{f^{(m)}\left(x^{(i)}\right)}, \quad i=0,1, \ldots,
$$

para o caso em que $x$ é uma raiz de multiplicidade $m$ de $f(x)$, Arthur [2] propôs o método abaixo.

Como $f^{(m)}(x) \neq 0$, existe uma vizinhança de $x$, tal que para qualquer $y$ nesta vizinhaça $f^{(m)}(y) \neq 0$. Assim, pode-se encontrar um intervalo $X^{(0)}$ tal que $0 \notin F^{(m)}\left(X^{(0)}\right)$. Definimos então, a função

$$
N(X)=m(X)-\frac{f^{(m-1)}(m(X))}{F^{(m)}(X)}
$$

e o método iterativo para zeros múltiplos:

$$
X^{(i+1)}=X^{(i)} \cap N\left(X^{(i)}\right) \quad, \quad i=0,1, \ldots
$$

\subsection{Observações}

\section{1) Teste de Parada:}

Para cada um dos métodos iterativos anteriores temos:

$$
X^{(i+1)}=N\left(X^{(i)}\right) \cap Y^{(i)}, \quad \text { onde } \quad X^{(i)} \subseteq Y^{(i)} \quad \text { e } x^{*} \in X^{(i)}, \quad i=0,1,2, \ldots
$$

Temos então, uma sequência de intervalos encaixantes $\left\{X^{(i)}\right\}_{i=0}^{\infty}$ $\operatorname{com} x^{*} \in X^{(i)}, i=0,1,2, \ldots$ e baseando-nos no teorema 1.4 e na definiçâo 1.22 , paramos o processo quando $X^{(i)}=X^{(i+1)}$.

2) Portanto, podemos limitar os auto-valores reais de uma matriz determinando o seu polinômio característico intervalar pelo métodos de LeverrierFaddeev Intervalar ou Danilevsky Intervalar e, partindo de um intervalo inicial que contém um auto-valor simples desta matriz, pelos métodos de Moore, Herzberger ou Hansen, encontramos um intervalo que contém este auto-valor, com diámetro tão pequeno quanto possível ou, ainda, partindo de uma intervalo que contém um único auto-valor de multiplicidade $m$,pelo método de Arthur podemos limitá-lo. 


\subsection{Exemplos}

Os métodos intervalares dados neste capitulo foram programados em linguagem PASCAL, submetidos a microcomputador da linha IBM-PC e muitos testes foram realizados.

Daremos agora, alguns exemplos, onde apresentaremos a matriz real testada, seus auto-valores e polinômio característico, os coeficientes $P^{(i)}$, $i=1, \ldots, n$, dos polinômios característicos intervalares obtidos pelos métodos intervalares de Leverrier-Faddeev e Danilevsky (quando este for possível de ser executado) e ainda, intervalos iniciais adequados à execução dos métodos de NewtonMoore, Herzberger, Hansen e Arthur, juntamente com os intervalos soluções e o número de iteraçôes realizadas para obtê-los. Estes intervalos soluções são as raízes do polinômio característico intervalar e contêm os auto-valores da matriz real dada.

As matrizes apresentadas nestes exemplos foram construídas segundo Linhares [9], que nos fornece uma maneira fácil de se obter matrizes reais cujos auto-valores são conhecidos. 
1)

matriz real : $\left(\begin{array}{rrrrr}14 & -9 & 13 & 2 & -18 \\ 2 & 3 & 13 & 2 & -18 \\ 2 & 12 & 4 & 2 & -18 \\ 2 & 12 & -3 & 9 & -18 \\ 2 & 12 & -3 & 4 & -13\end{array}\right)$

auto-valores: $\quad-9,2, \quad 5, \quad 7 \quad \mathrm{e} \quad 12$,

polinômio característico: $-x^{5}+17 x^{4}+7 x^{3}-1265 x^{2}+6162 x-7560$

\section{Método de Leverrier-Faddeev Intervalar}

$$
\begin{aligned}
& P^{(0)}=[1.00000000000000 \mathrm{e}+00,1.00000000000000 \mathrm{e}+00] \\
& P^{(1)}=[-1.70000000000000 \mathrm{e}+01,-1.70000000000000 \mathrm{e}+01] \\
& P^{(2)}=[-7.00000000000078 \mathrm{e}+00,-6.99999999999924 \mathrm{e}+00] \\
& P^{(3)}=[1.26499999999996 \mathrm{e}+03,1.26500000000004 \mathrm{e}+03] \\
& P^{(4)}=[-6.16200000000134 \mathrm{e}+03,-6.16199999999866 \mathrm{e}+03] \\
& P^{(5)}=[7.55999999994864 \mathrm{e}+03,7.56000000005138 \mathrm{e}+03]
\end{aligned}
$$

\begin{tabular}{|c|c|ccc|}
\hline \hline $\begin{array}{c}\text { Intervalo } \\
\text { inicial }\end{array}$ & Intervalo & \multicolumn{3}{|c|}{$n$ o de iteraçóes } \\
solução & Mo. & He. & Ha. \\
\hline$[-11,-6.3]$ & {$[-9.00000000000130 e+00,-8.99999999999870 e+00]$} & 7 & 6 & 6 \\
{$[-16,-6.3]$} & {$[-9.00000000000130 e+00,-8.99999999999870 e+00]$} & $*$ & 7 & 6 \\
{$[1.0,2.5]$} & {$[1.99999999996716 \epsilon+00,2.00000000003285 e+00]$} & 8 & 7 & 7 \\
{$[0.6,4.3]$} & {$[1.99999999996716 e+00,2.00000000003285 \epsilon+00]$} & $*$ & 8 & $*$ \\
{$[4.9,5.1]$} & {$[4.99999999989952 e+00,5.00000000010044 e+00]$} & 5 & 5 & \\
& {$[4.99999999989951 e+00,5.00000000010044 \epsilon+00]$} & & & 3 \\
{$[4.6,5.1]$} & {$[4.99999999989952 e+00,5.00000000010044 e+00]$} & $*$ & 7 & $*$ \\
{$[6.9,7.1]$} & {$[6.99999999992149 e+00,7.00000000007854 e+00]$} & 3 & 3 & \\
& {$[6.99999999992149 e+00,7.00000000007853 e+00]$} & & & 3 \\
{$[6.6,7.1]$} & {$[6.99999999992149 e+00,7.00000000007854 \epsilon+00]$} & $*$ & 7 & $*$ \\
{$[11.5,12.5]$} & {$[1.19999999999899 e+01,1.20000000000101 e+01]$} & 4 & 4 & 4 \\
{$[11.5,13.5]$} & {$[1.19999999999899 e+01,1.20000000000101 e+01]$} & $*$ & 7 & 7 \\
\hline \hline
\end{tabular}

Mo. : Método Newton-Moore

He. : Método de Herzberger

Ha. : Método de Hansen

* não foi possível executar o método para este intervalo inicial. 


\section{Método de Danilevsky Intervalar}

$$
\begin{aligned}
& P^{(0)}=[1.00000000000000 \mathrm{e}+00,1.00000000000000 \mathrm{e}+00] \\
& P^{(1)}=[-1.70000000000033 \mathrm{e}+01,-1.69999999999967 \mathrm{e}+01] \\
& P^{(2)}=[-7.00000000001956 \mathrm{e}+00,-6.99999999998044 \mathrm{e}+00] \\
& P^{(3)}=[1.26499999999974 \mathrm{e}+03,1.26500000000026 \mathrm{e}+03] \\
& P^{(4)}=[-6.16200000000264 \mathrm{e}+03,-6.16199999999736 \mathrm{e}+03] \\
& P^{(5)}=[7.55999999998936 \mathrm{e}+03,7.56000000001062 \mathrm{e}+03]
\end{aligned}
$$

\begin{tabular}{|c|c|ccc|}
\hline \hline $\begin{array}{c}\text { Intervalo } \\
\text { inicial }\end{array}$ & Intervalo & \multicolumn{3}{|c|}{$n$ ede iteraçóes } \\
solução & Mo. & He. & Ha. \\
\hline$[-11,-6.3]$ & {$[-9.00000000000176 e+00,-8.99999999999823 e+00]$} & 7 & 6 & 6 \\
{$[-16,-6.3]$} & {$[-9.00000000000176 e+00,-8.99999999999823 e+00]$} & $*$ & 7 & 6 \\
{$[1.0,2.5]$} & {$[1.99999999998960 e+00,2.00000000001038 e+00]$} & 7 & 6 & 7 \\
{$[0.6,4.3]$} & {$[1.99999999998960 e+00,2.00000000001038 e+00]$} & $*$ & 7 & $*$ \\
{$[4.9,5.1]$} & {$[4.99999999994094 e+00,5.00000000005910 e+00]$} & 4 & 4 & 4 \\
{$[4.6,5.1]$} & {$[4.99999999994094 e+00,5.00000000005910 e+00]$} & $*$ & 8 & $*$ \\
{$[6.9,7.1]$} & {$[6.99999999992960 e+00,7.00000000007036 e+00]$} & 4 & 4 & \\
& {$[6.99999999992960 e+00,7.00000000007037 e+00]$} & & & 4 \\
{$[6.6,7.1]$} & {$[6.99999999992959 e+00,7.00000000007037 e+00]$} & $*$ & 6 & $*$ \\
{$[11.5,12.5]$} & {$[1.19999999999753 e+01,1.20000000000247 e+01]$} & 4 & 4 & 4 \\
{$[11.5,13.5]$} & {$[1.19999999999753 e+01,1.20000000000247 e+01]$} & $*$ & 7 & 8 \\
\hline
\end{tabular}

Mo. : Método Newton-Moore

He. : Método de Herzberger

Ha. : Método de Hansen

* não foi possível executar o método para este intervalo inicial. 
2)

matriz real : $\left(\begin{array}{rrrrr}60 & 5 & 10 & 15 & -89 \\ 10 & 55 & 10 & 15 & -89 \\ 10 & 15 & 50 & 15 & -89 \\ 10 & 15 & 20 & 45 & -89 \\ 10 & 15 & 20 & 25 & -69\end{array}\right)$

auto-valores: $1,20,30,40$ e 50 ,

polinômio característico: $-x^{5}+141 x^{4}-7240 x^{3}+161100 x^{2}-1354000 x+1200000$

Método de Leverrier-Faddeev Intervalar

$$
\begin{aligned}
& P^{(0)}=[1.00000000000000 \mathrm{e}+00,1.00000000000000 \mathrm{e}+00] \\
& P^{(1)}=[-1.41000000000000 \mathrm{e}+02,-1.41000000000000 \mathrm{e}+02] \\
& P^{(2)}=[7.23999999999996 \mathrm{e}+03,7.24000000000004 \mathrm{e}+03] \\
& P^{(3)}=[-1.61100000000006 \mathrm{e}+05,-1.61099999999994 \mathrm{e}+05] \\
& P^{(4)}=[1.35399999999902 \mathrm{e}+06,1.35400000000098 \mathrm{e}+06] \\
& P^{(5)}=[-1.20000000015774 \mathrm{e}+06,-1.19999999984226 \mathrm{e}+06]
\end{aligned}
$$

\begin{tabular}{|c|c|c|c|c|}
\hline Intervalo & Intervalo & $n$ nஉ & Eiter & \\
\hline inicial & solução & Mo. & He. & \\
\hline$[0.2,4.0]$ & {$[9.99999999849258 e-01,1.00000000015074 e+00]$} & 6 & 5 & \\
\hline & {$[9.99999999849257 e-01,1.00000000015074 \epsilon+00]$} & & & 6 \\
\hline$(-2.0,5.0]$ & {$[9.99999999849258 \epsilon-01,1.00000000015074 \epsilon+00]$} & * & 6 & 7 \\
\hline$[19.8,20.2]$ & {$[1.99999999984196 e+01,2.00000000015804 e+01]$} & 6 & 6 & 5 \\
\hline$[19.4,20.2]$ & {$[1.99999999984196 e+01,2.00000000015804 e+01]$} & * & 8 & $*$ \\
\hline$[29.99,30.01]$ & {$[2.99999999966582 e+01,3.00000000033418 \epsilon+01]$} & 4 & 4 & \\
\hline & {$[2.99999999966581 e+01,3.00000000033418 \epsilon+01]$} & & & 3 \\
\hline$[39.99,40.01]$ & {$[3.99999999973136 e+01,4.00000000026867 \epsilon+01]$} & 3 & 3 & \\
\hline & {$[3.99999999973134 e+01,4.00000000026864 \epsilon+01]$} & & & 5 \\
\hline$[49.9,50.2]$ & {$[4.99999999992258 e+01,5.00000000007744 \epsilon+01]$} & 7 & 7 & \\
\hline & {$[4.99999999992256 e+01,5.00000000007744 \epsilon+01]$} & & & 7 \\
\hline$[49.9,50.5]$ & {$[4.99999999992254 e+01,5.00000000007745 e+01]$} & * & 7 & * \\
\hline
\end{tabular}

Mo. : Método Newton-Moore

He. : Método de Herzberger

Ha. : Método de Hansen

* não foi possível executar o método para este intervalo inicial. 


\section{Método de Danilevsky Intervalar}

$$
\begin{aligned}
& P^{(0)}=[1.00000000000000 \mathrm{e}+00,1.00000000000000 \mathrm{e}+00] \\
& P^{(1)}=[-1.41000000000055 \mathrm{e}+02,-1.40999999999945 \mathrm{e}+02] \\
& P^{(2)}=[7.23999999999542 \mathrm{e}+03,7.24000000000458 \mathrm{e}+03] \\
& P^{(3)}=[-1.61100000000134 \mathrm{e}+05,-1.61099999999866 \mathrm{e}+05] \\
& P^{(4)}=[1.35399999999864 \mathrm{e}+06,1.35400000000137 \mathrm{e}+06] \\
& P^{(5)}=[-1.20000000001242 \mathrm{e}+06,-1.19999999998764 \mathrm{e}+06]
\end{aligned}
$$

\begin{tabular}{|c|c|ccc|}
\hline \hline $\begin{array}{c}\text { Intervalo } \\
\text { inicial }\end{array}$ & Intervalo & \multicolumn{3}{|c|}{$n$ - de iteraçóes } \\
solução & Mo. & He. & Ha. \\
\hline$[0.2,4.0]$ & {$[9.99999999986832 e-01,1.00000000001322 e+00]$} & 7 & 5 & 7 \\
{$[-2.0,5.0]$} & {$[9.99999999986832 e-01,1.00000000001322 e+00]$} & $*$ & 7 & $*$ \\
{$[19.8,20.2]$} & {$[1.99999999987832 e+01,2.00000000012168 e+01]$} & 5 & 5 & 6 \\
{$[19.4,20.2]$} & {$[1.99999999987833 e+01,2.00000000012167 e+01]$} & $*$ & 10 & $*$ \\
{$[29.99,30.01]$} & {$[2.99999999941002 e+01,3.00000000058990 e+01]$} & 4 & 4 & \\
& {$[2.99999999941005 e+01,3.00000000058991 e+01]$} & & & 4 \\
{$[39.99,40.01]$} & {$[3.99999999908277 e+01,4.00000000091738 e+01]$} & 3 & 3 & \\
& {$[3.99999999908274 e+01,4.00000000091735 e+01]$} & & & 7 \\
{$[49.9,50.2]$} & {$[4.99999999954675 e+01,5.00000000045321 e+01]$} & & 6 & 8 \\
& {$[4.99999999954673 e+01,5.00000000045319 e+01]$} & 6 & & \\
{$[49.9,50.5]$} & {$[4.99999999954675 e+01,5.00000000045218 e+01]$} & $*$ & 8 & $*$ \\
\hline \hline
\end{tabular}

Mo. : Método Newton-Moore

He. : Método de Herzberger

Ha. : Método de Hansen

* não foi possivel executar o método para este intervalo inicial. 
3)

matriz real : $\left(\begin{array}{lll}5 & 1 & 1 \\ 2 & 4 & 1 \\ 2 & 2 & 3\end{array}\right)$

auto-valores: $2, \quad 3$ e 7 ,

polinômio característico: $-x^{3}+12 x^{2}-41 x+42$

\section{Método de Leverrier-Faddeev Intervalar}

$$
\begin{aligned}
& P^{(0)}=[1.00000000000000 \mathrm{e}+00,1.00000000000000 \mathrm{e}+00] \\
& P^{(1)}=[-1.20000000000000 \mathrm{e}+01,-1.20000000000000 \mathrm{e}+01] \\
& P^{(2)}=[4.09999999999999 \mathrm{e}+01,4.10000000000001 \mathrm{e}+01] \\
& P^{(3)}=[-4.20000000000006 \mathrm{e}+01,-4.19999999999994 \mathrm{e}+01]
\end{aligned}
$$

\begin{tabular}{|c|c|ccc|}
\hline \hline $\begin{array}{c}\text { Intervalo } \\
\text { inicial }\end{array}$ & $\begin{array}{c}\text { Intervalo } \\
\text { soluçâo }\end{array}$ & \multicolumn{3}{|c|}{$n$ o. de iteraçóes } \\
Mo. & He. & Ha. \\
\hline$[1.8,2.2]$ & {$[1.99999999999985 e+00,2.00000000000015 e+00]$} & 4 & 4 & 3 \\
{$[1.8,2.6]$} & {$[1.99999999999985 e+00,2.00000000000015 e+00]$} & $*$ & 7 & $*$ \\
{$[2.8,3.1]$} & {$[2.99999999999978 e+00,3.00000000000022 \epsilon+00]$} & 7 & 7 & 7 \\
{$[2.8,3.4]$} & {$[2.99999999999977 e+00,3.00000000000022 \epsilon+00]$} & $*$ & 8 & $*$ \\
{$[6.4,8.2]$} & {$[6.99999999999992 e+00,7.00000000000008 e+00]$} & 7 & 6 & 7 \\
{$[6.2,8.7]$} & {$[6.99999999999992 e+00,7.00000000000008 \epsilon+00]$} & $*$ & 7 & 7 \\
{$[6.0,20.0]$} & {$[6.99999999999992 e+00,7.00000000000008 \epsilon+00]$} & $*$ & $*$ & 8 \\
\hline
\end{tabular}

Mo. : Método Newton-Moore

He. : Método de Herzberger

Ha. : Método de Hansen

* não foi possível executar o método para este intervalo inicial. 


\section{Método de Danilevsky Intervalar}

$$
\begin{aligned}
& P^{(0)}=[1.00000000000000 \mathrm{e}+00,1.00000000000000 \mathrm{e}+00] \\
& P^{(1)}=[-1.20000000000000 \mathrm{e}+01,-1.20000000000000 \mathrm{e}+01] \\
& P^{(2)}=[4.09999999999999 \mathrm{e}+01,4.10000000000001 \mathrm{e}+01] \\
& P^{(3)}=[-4.20000000000002 \mathrm{e}+01,-4.19999999999998 \mathrm{e}+01]
\end{aligned}
$$

\begin{tabular}{|c|c|ccc|}
\hline \hline $\begin{array}{c}\text { Intervalo } \\
\text { inicial }\end{array}$ & Intervalo & \multicolumn{3}{|c|}{$n$ o de iteraçóes } \\
\hline$[1.8,2.2]$ & {$[1.99999999999991 e+00,2.00000000000009 e+00]$} & 3 & 3 & \\
& {$[1.99999999999990 e+00,2.00000000000010 e+00]$} & & & 3 \\
{$[1.8,2.6]$} & {$[1.99999999999991 e+00,2.00000000000009 e+00]$} & $*$ & 7 & $*$ \\
{$[2.8,3.1]$} & {$[2.99999999999983 e+00,3.00000000000017 e+00]$} & 7 & 6 & 7 \\
{$[2.8,3.4]$} & {$[2.99999999999983 e+00,3.00000000000017 e+00]$} & $*$ & 7 & $*$ \\
{$[6.4,8.2]$} & {$[6.99999999999991 e+00,7.00000000000009 e+00]$} & 7 & 7 & 7 \\
{$[6.2,8.7]$} & {$[6.99999999999991 e+00,7.00000000000009 e+00]$} & $*$ & 7 & 7 \\
{$[6.0,20.0]$} & {$[6.99999999999991 e+00,7.00000000000009 e+00]$} & $*$ & $*$ & 8 \\
\hline \hline
\end{tabular}

Mo. : Método Newton-Moore

He. : Método de Herzberger

Ha. : Método de Hansen

* não foi possível executar o método para este intervalo inicial. 
4)

matriz real : $\left(\begin{array}{rrrrrr}6 & -10 & 14 & -5 & -7 & 4 \\ 2 & -6 & 14 & -5 & -7 & 4 \\ 2 & 2 & 6 & -5 & -7 & 4 \\ 2 & 2 & -1 & 2 & -7 & 4 \\ 2 & 2 & -1 & -3 & -2 & 4 \\ 2 & 2 & -1 & -3 & -1 & 3\end{array}\right)$

auto-valores: $\quad-8, \quad-1, \quad 2, \quad 4, \quad 5$ e 7 ,

polinômio característico: $x^{6}-9 x^{5}-39 x^{4}+585 x^{3}-1554 x^{2}+72 x+2240$

Método de Leverrier-Faddeev Intervalar

$$
\begin{aligned}
& P^{(0)}=[1.00000000000000 \mathrm{e}+00,1.00000000000000 \mathrm{e}+00] \\
& P^{(1)}=[-9.00000000000001 \mathrm{e}+00,-8.99999999999999 \mathrm{e}+00] \\
& P^{(2)}=[-3.90000000000003 \mathrm{e}+01,-3.89999999999997 \mathrm{e}+01] \\
& P^{(3)}=[5.84999999999993 \mathrm{e}+02,5.85000000000007 \mathrm{e}+02] \\
& P^{(4)}=[-1.55400000000016 \mathrm{e}+03,-1.55399999999984 \mathrm{e}+03] \\
& P^{(5)}=[7199999999961949 \mathrm{e}+01,7.20000000038087 \mathrm{e}+01] \\
& P^{(6)}=[2.23999999990983 \mathrm{e}+03,2.24000000009016 \mathrm{e}+03]
\end{aligned}
$$

\begin{tabular}{|c|c|ccc|}
\hline Intervalo & Intervalo & \multicolumn{3}{|c|}{$n$ od de iterações } \\
inicial & solução & Mo. & He. & Ha. \\
\hline$[-10.0,-6.8]$ & {$[-8.00000000000083 e+00,-7.99999999999917 e+00]$} & 7 & 7 & 6 \\
{$[-9.5,-4.0]$} & {$[-8.00000000000083 e+00,-7.99999999999917 e+00]$} & $*$ & 8 & $*$ \\
{$[-4.0,0.0]$} & {$[-1.00000000001868 \epsilon+00,-9.99999999981319 e-01]$} & 8 & 7 & 7 \\
{$[1.9,2.2]$} & {$[1.99999999989056 e+00,2.00000000010944 e+00]$} & 8 & 7 & 7 \\
{$[1.6,2.5]$} & {$[1.99999999989056 e+00,2.00000000010944 e+00]$} & $*$ & 8 & $*$ \\
{$[3.99,4.01]$} & {$[3.99999999969850 e+00,4.00000000030145 e+00]$} & 4 & 4 & \\
& {$[3.99999999969850 e+00,4.00000000030146 e+00]$} & & & 5 \\
{$[4.99,5.01]$} & {$[4.99999999975565 e+00,5.00000000024440 e+00]$} & 4 & 4 & \\
& {$[4.99999999975565 \epsilon+00,5.00000000024439 e+00]$} & & & 4 \\
{$[4.99,5.1]$} & {$[4.99999999975565 e+00,5.00000000024439 e+00]$} & $*$ & 6 & $*$ \\
{$[6.9,7.1]$} & {$[6.99999999996442 e+00,5.00000000003556 \epsilon+00]$} & 5 & 5 & 5 \\
{$[6.9,7.3]$} & {$[6.99999999996442 e+00,5.00000000003556 e+00]$} & $*$ & 6 & $*$ \\
\hline \hline
\end{tabular}

Mo. : Método Newton-Moore

He. : Método de Herzberger

Ha. : Método de Hansen

* não foi possível executar o método para este intervalo inicial. 
5)

matriz real : $\left(\begin{array}{rrrrr}5 & 1 & 6 & 2 & -11 \\ 2 & 4 & 6 & 2 & -11 \\ 2 & 1 & 9 & 2 & -11 \\ 2 & 1 & 6 & 5 & -11 \\ 2 & 1 & 6 & 2 & -8\end{array}\right)$

auto-valores: $3, \quad 3, \quad 3, \quad 3$ e 3 ,

polinômio característico: $-x^{5}+15 x^{4}-90 x^{3}+270 x^{2}-405 x+243$

Método de Leverrier-Faddeev Intervalar

$$
\begin{aligned}
& P^{(0)}=[1.00000000000000 \mathrm{e}+00,1.00000000000000 \mathrm{e}+00] \\
& P^{(1)}=[-1.50000000000000 \mathrm{e}+01,-1.50000000000000 \mathrm{e}+01] \\
& P^{(2)}=[8.99999999999996 \mathrm{e}+01,9.00000000000004 \mathrm{e}+01] \\
& P^{(3)}=[-2.70000000000009 \mathrm{e}+02,-2.69999999999991 \mathrm{e}+02] \\
& P^{(4)}=[4.04999999999808 \mathrm{e}+02,4.05000000000192 \mathrm{e}+02] \\
& P^{(5)}=[-2.43000000004303 \mathrm{e}+02,-2.42999999995697 \mathrm{e}+02]
\end{aligned}
$$

\begin{tabular}{|c|c|cc|}
\hline \hline $\begin{array}{c}\text { Intervalo } \\
\text { inicial }\end{array}$ & $\begin{array}{c}\text { Intervalo } \\
\text { solução }\end{array}$ & $\begin{array}{c}\text { Arthus } \\
n \text { ode iteraçōes }\end{array}$ \\
\hline$[0.0,6.0]$ & {$[2.999999999999999 e+00,3.00000000000001 \epsilon+00]$} & 4 & 5 \\
{$[2.0,4.0]$} & {$[2.99999999999999 \epsilon+00,3.00000000000001 e+00]$} & 4 & 5 \\
{$[-2.0,8.0]$} & {$[2.99999999999999 e+00,3.00000000000001 e+00]$} & 4 & 5 \\
\hline
\end{tabular}

$m$ : multiplicidade do auto-valor. 
6)

matriz real : $\left(\begin{array}{rrrr}4 & 12 & 2 & -6 \\ 7 & 9 & 2 & -6 \\ 7 & 2 & 9 & -6 \\ 7 & 2 & 2 & 1\end{array}\right)$

auto-valores: $\quad-3,7, \quad 7 \quad$ e 12 ,

polinômio característico: $x^{4}-23 x^{3}+139 x^{2}+63 x-1764$

Método de Leverrier-Faddeev Intervalar

$$
\begin{aligned}
& P^{(0)}=[1.00000000000000 \mathrm{e}+00,1.00000000000000 \mathrm{e}+00] \\
& P^{(1)}=[-2.30000000000000 \mathrm{e}+01,-2.30000000000000 \mathrm{e}+01] \\
& P^{(2)}=[1.39000000000000 \mathrm{e}+02,1.39000000000000 \mathrm{e}+02] \\
& P^{(3)}=[6.29999999999925 \mathrm{e}+01,6.30000000000075 \mathrm{e}+01] \\
& P^{(4)}=[-1.76400000000016 \mathrm{e}+03,-1.76399999999984 \mathrm{e}+03]
\end{aligned}
$$

\begin{tabular}{|c|c|c|c|}
\hline $\begin{array}{c}\text { Intervalo } \\
\text { inicial }\end{array}$ & $\begin{array}{c}\text { Intervalo } \\
\text { solução }\end{array}$ & \multicolumn{2}{|c|}{$\begin{array}{c}\text { Arthur } \\
n \text { e de iterações }\end{array}$} \\
\hline$[6.4,7.6]$ & {$[6.99999999999970 e+00,7.00000000000030 e+00]$} & 4 & 2 \\
{$[6.5,7.5]$} & {$[6.99999999999971 e+00,7.00000000000030 e+00]$} & 4 & 2 \\
\hline
\end{tabular}

m: multiplicidade do auto-valor.

\begin{tabular}{|c|c|ccc|}
\hline \hline Intercalo & Intervalo & \multicolumn{3}{|c|}{$n$ de iteraçóes } \\
inicial & soluçâo & Mo. & He. & Ha. \\
\hline$[-10.0,-1.0]$ & {$[-3.00000000000013 e+00,-2.99999999999987 e+00]$} & 7 & 7 & 6 \\
{$[11.4,12.1]$} & {$[1.19999999999991 e+01,1.20000000000009 e+01]$} & 6 & 6 & 5 \\
{$[10.7,13.2]$} & {$[1.19999999999991 e+01,1.20000000000009 e+01]$} & $*$ & 7 & $*$ \\
\hline
\end{tabular}

Mo. : Método Newton-Moore

He. : Método de Herzberger

Ha. : Método de Hansen

* não foi possível executar o método para este intervalo inicial. 


\section{Capítulo 4}

\section{Versão Intervalar de Métodos Iterativos para a Determinação de Auto-valores de Matrizes}

Neste capítulo desenvolveremos versões intervalares dos métodos iterativos LR de Rutishauser e QR de Francis, para a solução do problema de auto-valores de matrizes.

\subsection{Método LR de Rutishauser}

Método $L R$ no caso real:

Sejam $\mathcal{A}$ uma matriz real, $n \times n$, e $M_{i}$ seus menores principais de ordem $i, i=1, \ldots, n$. Se $\operatorname{det} M_{i} \neq 0$ para $i=1, \ldots, n$, então existe uma única decomposição $\mathcal{A}=L R$, onde $L$ é uma matriz triangular inferior com todos os elementos da diagonal principal iguais a 1 e $R$ é uma matriz triangular superior.

Usando o resultado acima, constrói-se o método iterativo conhecido por Método LR ou de Rutishauser, definindo-se uma sequência de matrizes $\mathcal{A}^{(k)}$, onde $\mathcal{A}^{(1)}=\mathcal{A}$ (matriz original) e $\mathcal{A}^{(k+1)}$ é obtida decompondo-se a matriz $\mathcal{A}^{(k)}$ no produto $\mathcal{A}^{(k)}=L^{(k)} R^{(k)}$ dado acima, e multiplicando as matrizes obtidas em ordem inversa, ou seja: 
- $\mathcal{A}^{(1)}=\mathcal{A}$

- para $k=1,2, \ldots$ repita

$$
\begin{aligned}
& \mathcal{A}^{(k)}=L^{(k)} R^{(k)} \\
& \mathcal{A}^{(k+1)}=R^{(k)} L^{(k)}
\end{aligned}
$$

Prova-se que $\mathcal{A}^{(k)}$ e $\mathcal{A}^{(k+1)}$ são matrizes similares e portanto têm os mesmos auto-valores.

Prova-se também que se certas condições são impostas à matriz $\mathcal{A}$, $\lim _{k \rightarrow \infty} \mathcal{A}^{(k)}=\mathcal{A}^{(\infty)}$, onde $\mathcal{A}^{(\infty)}$ é uma matriz triangular superior, ao longo de cuja diagonal principal estão os auto-valores de $\mathcal{A}$, dispostos em ordem decrescente de seus módulos. Ver [15] e [16].

\section{Método $L R$ no caso intervalar:} e $A_{i j}=\left[a_{i j}, a_{i j}\right]$.

Seja $\mathcal{A}=\left(a_{i j}\right)$ matriz real de ordem $n$ e $[A]=\mathcal{A}$, isto é, $[A]=\left(A_{i j}\right)$

Fizemos a versão intervalar direta do método LR, isto é, substituímos todas as operações e matrizes reais por operações e matrizes intervalares, respectivamente, construindo uma sequencia de matrizes intervalares $\left[A^{(k)}\right]$ que converge para uma matriz triangular superior intervalar cujos intervalos da diagonal principal contêm os auto-valores da matriz original $\mathcal{A}$, da seguinte forma:

- $\left[A^{(1)}\right]=[A]$

- para $k=1,2, \ldots$ repita

$$
\begin{aligned}
& {\left[A^{(k)}\right]=\left[L^{(k)}\right]\left[R^{(k)}\right]} \\
& {\left[A^{(k+1)}\right]=\left[R^{(k)}\right]\left[L^{(k)}\right]}
\end{aligned}
$$

onde $\left[L^{(k)}\right]$ é matriz triangular inferior intervalar, cujos elementos da diagonal principal são iguais a $[1,1]$ e $\left[R^{(k)}\right]$ é matriz triangular superior intervalar.

O método, no caso real, possui a característica de convergir para uma matriz triangular superior em cuja diagonal principal estão os auto-valores da matriz inicial dispostos em ordem decrescente de seus módulos. Para isto, no decorrer do método, os auto-valores que não estão no seu devido lugar vâo sendo ordenados. 
No caso intervalar, o mesmo acontece, mas quando os intervalos diagonais vão sendo ordenados, pode ocorrer divisão por intervalo que contém o zero, e então não podemos mais continuar a execução do método.

Experimentalmente, concluímos que o método intervalar funciona perfeitamente bem quando a matriz original satisfaz as condiçōes de convergência do método real e não houver a necessidade de reordenação dos auto-valores ao longo da diagonal principal das matriz triangulares superiores geradas. Verifcamos que não há necessidade de reordenação dos auto-valores quando, a cada passo, os menores principais das matrizes $\left[A^{(k)}\right],\left(\Delta_{i}, i=0,1, \ldots, n, \Delta_{0}=1\right)$ satisfizerem:

$$
\left(\Delta_{i}\right)^{2}>\left|\Delta_{i-1} * \Delta_{i+1}\right|, \quad i=1,2, \ldots, n-1,
$$

ou ainda se,

$$
\left|r_{i i 1}^{(k)}\right|<\left|r_{i-1, i-1,1}^{(k)}\right|, \quad i=2,3, \ldots, n,
$$

onde $\left[R^{(k)}\right]=\left(R_{i j}^{(k)}\right)$ e $\quad\left(R_{i i}^{(k)}\right)=\left[r_{i i 1}^{(k)}, r_{i i 2}^{(k)}\right]$.

\section{Algoritmo Intervalar}

Dada $\mathcal{A}=\left(a_{i j}\right)$ - uma matriz real de ordem $n$.

1. Definir a matriz intervalar pontual

$$
[A]=\mathcal{A} \text { onde }[\stackrel{A}{A}]=\left(A_{i j}\right) \text { e } \quad A_{i j}=\left[a_{i j}, a_{i j}\right]
$$

2. Fazer $\left[A^{(1)}\right]=[A]$

3. Para $k=1,2, \ldots$, executar os passos abaixo até que $A_{i i}^{(k)}=A_{i i}^{(k+1)}$, $i=1, \ldots, n$

(a) Decompor $\left[A^{(k)}\right]=\left[L^{(k)}\right]\left[R^{(k)}\right]$

(b) Fazer $[B]=\left[R^{(k)}\right]\left[L^{(k)}\right]$

(c) Para $i, j=1,2, \ldots, n$, se $A_{i j}^{(k)} \subset B_{i j}$ então $A_{i j}^{(k+1)}=A_{i j}^{(k)}$
senão $A_{i j}^{(k+1)}=B_{i j}$

4. Fim do algoritmo. 


\subsection{Método QR de Francis}

\section{Método QR, no caso real} de matrizes consiste em:

O método iterativo de Francis para a determinação dos autovalores

Dada a matriz $\mathcal{A}$, define-se uma sequência de matrizes $\mathcal{A}^{(k)}$, onde $\mathcal{A}^{(1)}=\mathcal{A}$ (matriz original), e $\mathcal{A}^{(k+1)}$ é obtida de $\mathcal{A}^{(k)}$, decompondo-a no produto $\mathcal{A}^{(k)}=Q^{(k)} R^{(k)}$, onde $Q^{(k)}$ é uma matriz ortogonal e $R^{(k)}$ é uma matriz triangular superior com os elementos da diagonal principal positivos, e efetuando o produto em ordem inversa, isto é:

- $\mathcal{A}^{(1)}=\mathcal{A}$

- para $k=1,2, \ldots$ repita

$$
\begin{aligned}
& \mathcal{A}^{(k)}=Q^{(k)} R^{(k)} \\
& \mathcal{A}^{(k+1)}=R^{(k)} Q^{(k)}
\end{aligned}
$$

Verifica-se facilmente, que o método acima consiste de uma série de transformações de similaridade aplicadas à matriz inicial $\mathcal{A}$ :

$\mathcal{A}^{(k)}=Q^{(k)} R^{(k)} \Rightarrow\left(Q^{(k)}\right)^{-1} \mathcal{A}^{(k)}=R^{(k)} \quad$ e $\quad \mathcal{A}^{(k+1)}=R^{(k)} Q^{(k)}=\left(Q^{(k)}\right)^{-1} \mathcal{A}^{(k)} Q^{(k)}$. Portanto, $\mathcal{A}^{(k+1)}$ e $\mathcal{A}^{(k)}$ são similires.

Francis provou que se certas condições são impostas à matriz $\mathcal{A}$, $\lim _{k \rightarrow \infty} \mathcal{A}^{(k)}=\mathcal{A}^{(\infty)}$, onde $\mathcal{A}^{(\infty)}$ é uma matriz triangular superior, ao longo de cuja diagonal estâo os auto-valores de $\mathcal{A}$, dispostos em ordem decrescente de seus módulos. Ver [6], [15] e [16].

\section{Método QR, no caso intervalar}

anterior.

Seja $\mathcal{A}=\left(a_{i j}\right)$ matriz real de ordem $n$ e $[A]=\mathcal{A}$, como no método

Construímos, a partir de $[A]$, uma sequência de matrizes intervalares $\left[A^{(k)}\right], k=1,2, \ldots$, da seguinte forma: 
- $\left[A^{(1)}\right]=[A]$

- para $k=1,2, \ldots$ repita

$\left[A^{(k)}\right]=\left[Q^{(k)}\right]\left[R^{(k)}\right]$

$\left[A^{(k+1)}\right]=\left[R^{(k)}\right]\left[Q^{(k)}\right]$

onde $\left[R^{(k)}\right]$ é matriz triangular superior intervalar e $\left[Q^{(k)}\right]$ é matriz ortogonal intervalar.

Obtemos melhores resultados, quando ao fazermos a decomposição intervalar $\left[A^{(k)}\right]=\left[Q^{(k)}\right]\left[R^{(k)}\right]$ evitamos o uso de raizes quadradas, isto porque, pode ocorrer o caso do intervalo radicando conter números negativos, devido aos arredondamentos efetuados.

Observamos que se os auto-valores da matriz original são distintos em módulo, o método QR intervalar converge para uma matriz triangular superior intervalar, ao longo de cuja diagonal principal estão intervalos que contêm os auto-valores de $\mathcal{A}$.

\section{Algoritmo Intervalar}

Dada $\mathcal{A}=\left(a_{i j}\right)$ - uma matriz real de ordem $n$.

1. Definir a matriz intervalar pontual

$$
[\stackrel{A}{]}]=\mathcal{A} \quad \text { onde }[\stackrel{A}{A}]=\left(A_{i j}\right) \text { e } \quad A_{i j}=\left[a_{i j}, a_{i j}\right]
$$

2. Fazer $\left[A^{(1)}\right]=[A]$

3. Para $k=1,2, \ldots$, executar os passos abaixo até que $A_{i i}^{(k)}=A_{i i}^{(k+1)}$, $i=1, \ldots, n$

(a) Decompor $\left[A^{(k)}\right]=\left[Q^{(k)}\right]\left[R^{(k)}\right]$

(b) Fazer $[B]=\left[R^{(k)}\right]\left[Q^{(k)}\right]$

(c) Para $i, j=1,2, \ldots, n$, se $A_{i j}^{(k)} \subset B_{i j}$

$$
\begin{aligned}
& \text { então } A_{i j}^{(k+1)}=A_{i j}^{(k)} \\
& \text { senão } A_{i j}^{(k+1)}=B_{i j}
\end{aligned}
$$

4. Fim do algoritmo. 


\subsection{Observações}

\section{1) Teste de Parada:}

Como, para os métodos descritos acima, não utilizamos dados inicjais que contêm os dados exatos, não podemos fazer interseção do tipo $\left[A^{(k+1)}\right]=\left[A^{(k)}\right] \cap\left[R^{(k)}\right]\left[L^{(k)}\right]$ ou $\left[A^{(k+1)}\right]=\left[A^{(k)}\right] \cap\left[R^{(k)}\right]\left[Q^{(k)}\right]$ para $k=0,1,2, \ldots$ $A_{i j}^{(k)} \subset A_{i j}^{(k+1)}, i, j=1, \ldots, n$.

Fazemos então a intersecção $A_{i j}^{(k+1)}=A_{i j}^{(k)} \cap A_{i j}^{(k+1)}$ apenas quando E paramos o método quando $A_{i i}^{(k+1)}=A_{i i}^{(k)}, i=1, \ldots, n$.

2) Notamos que nos métodos iterativos dados acima, a sequência de matrizes $\left[A^{(k)}\right]$ vai se aproximando de uma matriz intervalar ao longo de cuja diagonal principal estão intervalos que contêm os auto-valores da matriz original, e que para algum $k=k_{0}>0, \quad\left[A^{\left(k_{0}\right)}\right]$ é exatamente esta matriz. As matrizes seguintes $\left[A^{\left(k_{0}+1\right)}\right],\left[A^{\left(k_{0}+2\right)}\right], \ldots$, têm em suas diagonais principais, intervalos que contêm os auto-valores de $A$, mas com diâmetro cada vez maior, isto por causa dos arredondamentos efetuados. Daí, a necessidade de se fazer intersecçōes entre os elementos das duas matrizes $\left[A^{(k)}\right]$ e $\left[A^{(k+1)}\right]$, para evitarmos acúmulo de erros desnecessários.

3) Observamos que os intervalos da diagonal principal contêm os auto-valores da matriz inicial e que o diâmetro destes intervalos são arbitrariamente pequenos, o que mostra que os métodos LR e QR intervalares obtém os auto-valores de $\mathcal{A}$, com alta precisão (veja exemplos).

\subsection{Exemplos}

As versões intervalares dos métodos $L R$ e $Q R$ foram implementadas em linguagem PASCAL e vários exemplos foram testados em microcomputador compativel com IBM-PC.

Aqui apresentamos algumas das matrizes testadas, juntamente com os intervalos resultantes que contêm os auto-valores obtidos pelas versões intervalares.

As matrizes apresentadas nestes exemplos também foram construídas segundo Linhares [9]. 
1)

matriz real : $\left(\begin{array}{rrrrr}14 & -9 & 13 & 2 & -18 \\ 2 & 3 & 13 & 2 & -18 \\ 2 & 12 & 4 & 2 & -18 \\ 2 & 12 & -3 & 9 & -18 \\ 2 & 12 & -3 & 4 & -13\end{array}\right)$

auto-valores: $\quad-9, \quad 2, \quad 5, \quad 7 \quad$ e 12 .

Obtemos os seguintes intervalos que limitam os auto-valores acima:

Método LR Intervalar com 39 iterações:

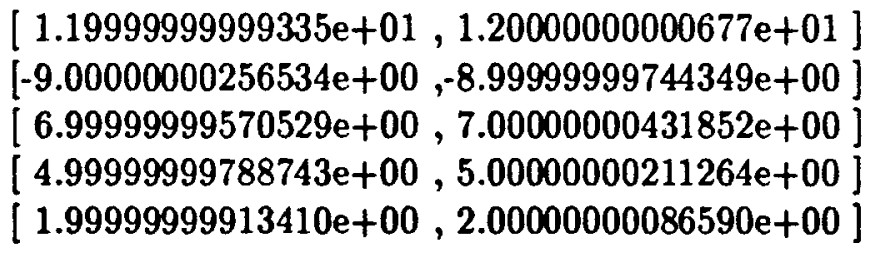

Método QR Intervalar com 38 iterações:

$\left[\begin{array}{l}1.19999999999931 \mathrm{e}+01,1.20000000000068 \mathrm{e}+00] \\ {[-9.00000000006230 \mathrm{e}+00,-8.99999999993757 \mathrm{e}+00]} \\ {[6.99999999991595 \mathrm{e}+00,7.00000000008403 \mathrm{e}+00]} \\ {[4.99999999995822 \mathrm{e}+00,5.00000000004157 \mathrm{e}+00]} \\ {[1.99999999998439 \mathrm{e}+00,2.00000000001549 \mathrm{e}+00]}\end{array}\right.$ 
2)

matriz real : $\left(\begin{array}{rrrrr}60 & 5 & 10 & 15 & -89 \\ 10 & 55 & 10 & 15 & -89 \\ 10 & 15 & 50 & 15 & -89 \\ 10 & 15 & 20 & 45 & -89 \\ 10 & 15 & 20 & 25 & -69\end{array}\right)$

auto-valores: $1, \quad 20,30,40$ e 50 .

Obtemos os seguintes intervalos que limitam os auto-valores acima:

Método LR Intervalar com 14 iterações:

$\left[\begin{array}{l}4.99999999999993 \mathrm{e}+01,5.00000000000007 \mathrm{e}+01 \\ {[3.99999999999990 \mathrm{e}+01,4.00000000000011 \mathrm{e}+01}\end{array}\right]$
$\left[\begin{array}{l}2.99999999999988 \mathrm{e}+01,3.0000000000012 \mathrm{e}+01 \\ {[1.99999999999988 \mathrm{e}+01,5.0000000000013 \mathrm{e}+01}\end{array}\right]$
$\left[\begin{array}{l}9.99999999999189 \mathrm{e}-01,2.00000000000076 \mathrm{e}+00 \\ {[}\end{array}\right]$

Método QR Intervalar com 14 iterações:

\footnotetext{
[ $4.99999999999958 \mathrm{e}+01,5.00000000000038 \mathrm{e}+01]$

[ $3.99999999999936 \mathrm{e}+01,4.00000000000062 \mathrm{e}+01]$

[2.99999999999923e+01, 3.00000000000076e+01]

$[1.99999999999932 \mathrm{e}+01,5.00000000000066 \mathrm{e}+01]$

$[9.99999999996328 \mathrm{e}-01,2.00000000000363 \mathrm{e}+00$ ]
} 
3)

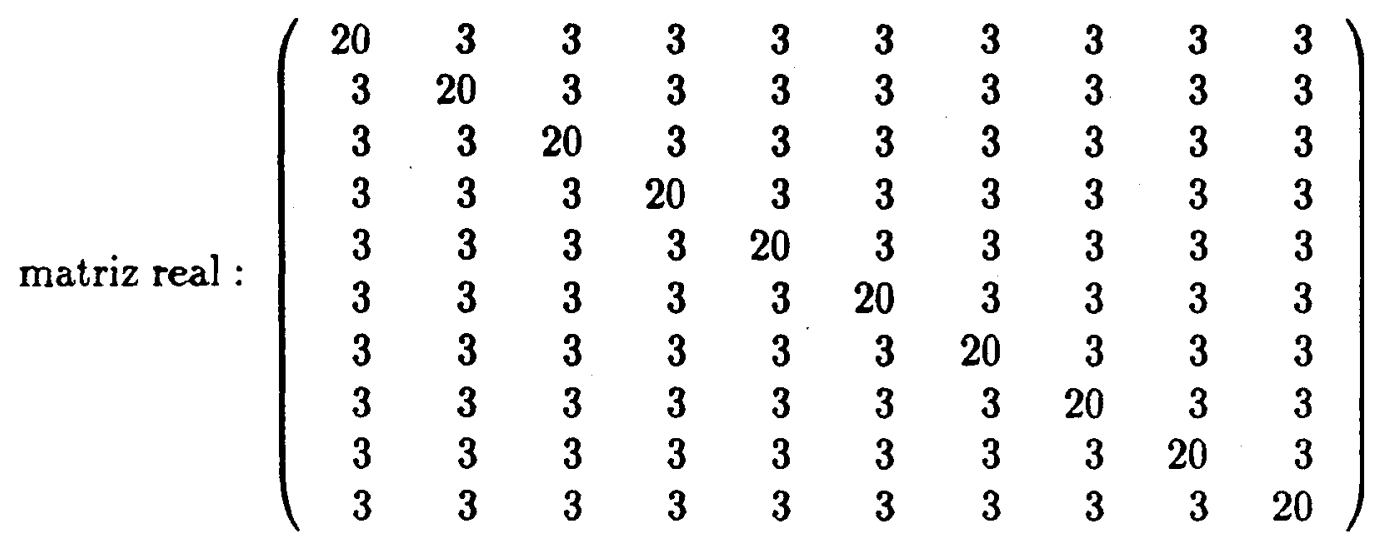

auto-valores: $17,17,17,17,17,17,17,17,17$ e 47.

Obtemos os seguintes intervalos que limitam os auto-valores acima:

Método LR Intervalar com 36 iterações:

$\left.\begin{array}{l}{\left[\begin{array}{l}4.69999999999959 \mathrm{e}+01,4.70000000000039 \mathrm{e}+01 \\ {[1.69999999999977 \mathrm{e}+01,1.70000000000024 \mathrm{e}+01}\end{array}\right]} \\ {\left[\begin{array}{l}1.69999999999982 \mathrm{e}+01,1.70000000000019 \mathrm{e}+01 \\ 1.69999999999984 \mathrm{e}+01,1.70000000000017 \mathrm{e}+01\end{array}\right]} \\ {\left[\begin{array}{l}1.69999999999985 \mathrm{e}+01,1.70000000000016 \mathrm{e}+01 \\ 1.69999999999986 \mathrm{e}+01,1.70000000000015 \mathrm{e}+01\end{array}\right]} \\ {\left[\begin{array}{l}1.69999999999987 \mathrm{e}+01,1.70000000000014 \mathrm{e}+01 \\ 1.69999999999987 \mathrm{e}+01,1.70000000000014 \mathrm{e}+01\end{array}\right]} \\ {[1.69999999999987 \mathrm{e}+01,1.70000000000014 \mathrm{e}+01} \\ 1.69999999999987 \mathrm{e}+01,1.70000000000013 \mathrm{e}+01\end{array}\right]$

Método QR Intervalar com 19 iterações:

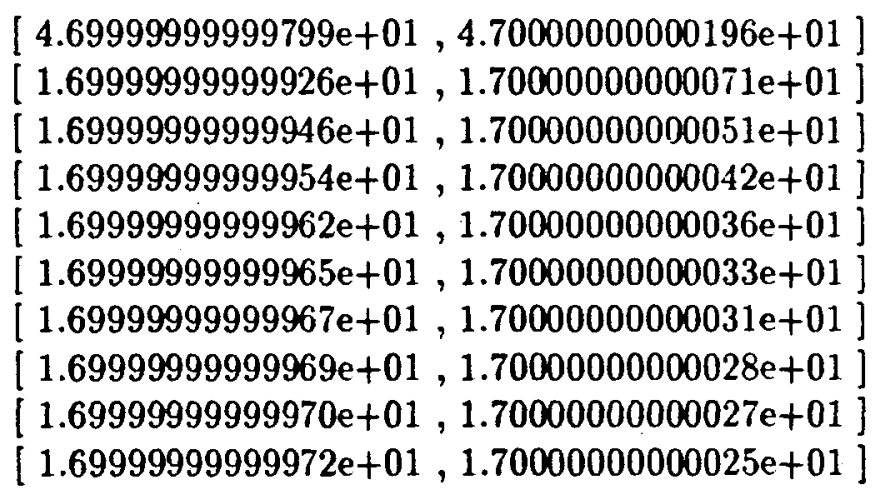


4)

matriz real : $\left(\begin{array}{rrrrrrrrrr}13 & -2 & 0 & 6 & 1 & -3 & -6 & 0 & 2 & -10 \\ 3 & 8 & 0 & 6 & 1 & -3 & -6 & 0 & 2 & -10 \\ 3 & -1 & 9 & 6 & 1 & -3 & -6 & 0 & 2 & -10 \\ 3 & -1 & 1 & 14 & 1 & -3 & -6 & 0 & 2 & -10 \\ 3 & -1 & 1 & 7 & 8 & -3 & -6 & 0 & 2 & -10 \\ 3 & -1 & 1 & 7 & 2 & 3 & -6 & 0 & 2 & -10 \\ 3 & -1 & 1 & 7 & 2 & -2 & -1 & 0 & 2 & -10 \\ 3 & -1 & 1 & 7 & 2 & -2 & -5 & 4 & 2 & -10 \\ 3 & -1 & 1 & 7 & 2 & -2 & -5 & 1 & 5 & -10 \\ 3 & -1 & 1 & 7 & 2 & -2 & -5 & 1 & 3 & -8\end{array}\right.$

auto-valores: $\quad 10,9, \quad 8, \quad 7, \quad 6, \quad 5, \quad 4, \quad 3, \quad 2 \quad$ e 1 .

Obtemos os seguintes intervalos que limitam os auto-valores acima:

Método LR Intervalar com 51 iterações:

[ 9.99999999999934e+00, $1.00000000000007 \mathrm{e}+01]$

$[8.99999999999935 \mathrm{e}+00,9.00000000000072 \mathrm{e}+00]$

$[7.99999999999918 \mathrm{e}+00,8.00000000000084 \mathrm{e}+00]$

$[6.99999999999841 \mathrm{e}+00,7.00000000000162 \mathrm{e}+00]$

$[5.99999999999820 \mathrm{e}+00,6.00000000000187 \mathrm{e}+00]$

$[4.99999999999802 \mathrm{e}+00,5.00000000000199 \mathrm{e}+00]$

$[3.99999999999787 \mathrm{e}+00,4.00000000000212 \mathrm{e}+00]$

$[2.99999999999766 \mathrm{e}+00,3.00000000000234 \mathrm{e}+00]$

$[1.99999999999742 \mathrm{e}+00,2.00000000000256 \mathrm{e}+00]$

$[9.99999999998028 \mathrm{e}-01,1.00000000000196 \mathrm{e}+00]$

Método QR Intervalar com 51 iteraçōes:

[ $9.99999999998385 \mathrm{e}+00,1.00000000000159 \mathrm{e}+01]$

[ 8.99999999999411e+00, 9.00000000000585e+00]

[ $7.99999999998263 \mathrm{e}+00,8.00000000001822 \mathrm{e}+00]$

$[6.99999999996463 \mathrm{e}+00,7.00000000003745 \mathrm{e}+00]$

[ $5.99999999996984 \mathrm{e}+00,6.00000000003033 \mathrm{e}+00]$

[ $4.99999999997239 \mathrm{e}+00,5.00000000002756 \mathrm{e}+00]$

[ 3.99999999997744e+00, 4.00000000002245e+00]

[ $2.99999999995489 \mathrm{e}+00,3.00000000004488 \mathrm{e}+00]$

$[1.99999999994557 \mathrm{e}+00,2.00000000005408 \mathrm{e}+00]$

[ $9.99999999965493 \mathrm{e}-01,1.00000000003433 \mathrm{e}+00$ ] 


\section{Capítulo 5}

\section{Conclusões}

Neste capítulo apresentaremos as conclusôes finais e algumas observações sobre os métodos numéricos intervalares construidos ou analisados.

Dos algoritmos construídos, o da versão intervalar do Método QR de Francis, foi o que apresentou melhor desempenho, funcionando tão bem quanto o do caso real, com as mesmas condiçōes de convergência deste caso. Nâo há restrições quanto a ordem (elevada ou não) da matriz. Como a extração de raiz quadrada, em Matemática Intervalar gera problemas, às vezes de difícil solução, optou-se, no cálculo das matrizes ortogonais intervalares $\left[Q^{(k)}\right], k=0,1,2, \ldots$, pela versão do método de Francis, que não faz uso dessa raiz. Assim, outras versōes do método $Q R$ foram descartadas, por terem apresentado problemas, dada a necessidade de extração de raizes quadradas. Aliás, há alguns tópicos que continuam a merecer a atenção dos pesquisadores em Análise Intervalar, pois estão ainda mal equacionados, como, por exemplo, o da função quadrada (cálculo do quadrado de um intervalo), o da extração de raiz quadrada e o cálculo de produto escalar de dois vetores intervalares.

A versão intervalar do método LR de Rutishauser apresenta, também, bom desempenho, funcionando tanto para matrizes de baixa, quanto de alta ordem e com as mesmas condiçôes de convergência do caso real. Há, porém, uma restrição importante: ocorrer a necessidade de reordenação dos auto-valores, ao longo da diagonal principal das matrizes triangulares superiores geradas. Verificou-se, experimentalmente que se as matrizes geradas $\left[A^{(k)}\right]$, forem tais que seus menores principais $\Delta_{i}, \quad i=0,1, \ldots, n \quad\left(\Delta_{0}=1\right)$ satisfizerem: $\left(\Delta_{j}\right)^{2}>\left|\Delta_{j-1} * \Delta_{j+1}\right|, \quad j=1,2, \ldots, n-1$, não ocorre a reordenação citada.

$\mathrm{Na}$ versão intervalar dos dois métodos iterativos citados, estabe- 
lecem-se, como teste de parada a condição que se interrompa a execução do problema quando se tiver

$$
A_{i i}^{(k+1)}=A_{i i}^{(k)}, \quad i=1,2, \ldots, n
$$

para alguma iteração $k=k_{0}>0$. Toma-se então, como os auto-valores intervalares de $\mathcal{A}$, os elementos diagonais de $\left[A^{k_{0}}\right]$.

Para os métodos iterativos QR e $L R$, várias versões intervalares foram tentadas. Apenas as apresentadas, neste trabalho, tiveram relativo sucesso. Também outros métodos iterativos mereceram nossa atenção, porém, sem sucesso. Vê-se, desse modo, que o assunto tem muito a oferecer, em termos de pesquisa.

A versão intervalar dos métodos de Leverrier-Faddeev e de Danilevsky (polinômio característico) funcionam bem apenas para matrizes de baixa ordem $n(n \leq 6)$. A partir desse valor, os intervalos obtidos continuam a conter os coeficientes do polinômio característico, porém com diâmetro crescendo muito com a ordem $n$. Mais um problema a merecer pesquisa posterior.

Quanto ao método de J. Zhinying para limitação de auto-valores e de auto-vetores de matriz $\mathcal{A}$ real, simétrica, observamos que, se os auto-vetores correspondentes ao auto-valor $\lambda \in \Lambda$ forem linearmente independentes, o comportamento é o mesmo que para matrizes simétricas.

No método de Zhiying quando obtemos duas iterações iguais, isto $\dot{e},\left[x_{i}^{(k)}\right]=\left[x_{i}^{(k+1)}\right]$ e temos que fazer bissecção no intervalo $\Lambda^{(k)}$ é interessante que recalculemos os vetores intervalares $\left[x_{i}\right]$ pelo método de Nickel a cada bissecção para se obter melhores limitantes para os auto-vetores.

Muitos problemas aqui citados e com os quais nos defrontamos no decorrer de nossa pesquisa derivam da não existência, no país, de máquina (computadores) com arquitetura ("hardware") adequadas ao processamento de dados intervalares. Tivemos que nos valer de microcomputadores da linha IBM-PC, utilizando a linguagem PASCAL munida de "software" especializado em Aritmética. Intervalar.

Uma das dificuldades por nós encontrada foi a escassa bibliografia disponível. Os artigos publicados sobre métodos intervalares para a solução do problema de auto-valores não passam de 04 ou 05 , o que uma vez mais, evidencia a complexidade do problema abordado. 


\section{Referências Bibliográficas}

[1] ALEFELD, G., HERZBERGER, J. Introduction to interval computations. New York, Academic Press, 1983.

[2] ARTHUR, D.W. The use of interval arithmetic to bound the zeros of real polynomials. J. Inst. Maths. Applics, v.10, p. 231-237, 1972.

[3] DEWAR, J.K.S. Procedures for interval arithmetic. Computer J., v.14, p. $447-450,1971$.

[4] DWYER, P.S. Linear computation. New York, John Wiley \& Sons, 1951.

[5] FADDEEVA, V.N. Computational methods of linear algebra. New York, Dover Publications, 1959.

[6] FRANCIS, J.F.G. The $Q R$ transformation. A unitary analogue to the $L R$ transformation, Parts I and II. Computer Journal, 4 p. 265-271; 332345, 1961/62.

[7] HANSEN, E. Interval forms of Newtons method. Computing, v.20, p. 153 163,1978 .

[8] HERZBERGER, J. Bemerkungen zu einem Verfahren von R. E. Moore. ZAMM, v.53, p.356-358, 1973.

[9] LINHARES, O.L. Classes de matrizes quadradas para teste de algoritmos computacionais. Notas do ICMSC - USP, v. 14, 1985.

[10] MOORE, R.E. Interval analysis. Englewood Cliffs, Prentice Hall, 1966.

[11] MOORE, R.E. Methods and applications of interval analysis. Philadelphia, SIAM, 1979.

[12] NICKEL, K.L.E. Bounding eigenvectors of a symmetric matrix. Interval 
Mathematics 1980, Academic Press, p. 433-447, 1980.

[13] QINGRONG, L., ZHIYING, J. A method for solving the eigenproblem of symmetric interval matrix. Freiburger Intervall - Berichte, v.87/7, p. 9-14, 1987.

[14] SCHWARZ, H.R., RUTISHAUSER, H., STIEFEL, E. Numerical analysis of symmetric matrices. Englewood Cliffs, Prentice-Hall, 1973.

[15] WILKINSON, J.H. The algebraic eigenvalue problems. Oxford, Claredon Press, 1965.

[16] WILKINSON, J.H. Convergence of the $L R, Q R$, and algorithms Computer Journal, v.8, p.77-84, 1965.

[17] ZHIYING, J. An interval method for bounding the eigenvalue and eigenvectors of a symmetric matrix. Freiburger Intervall - Berichte, v.87/7, p. $17-21,1987$. 